\title{
A mass-conservative characteristic splitting mixed element method for saltwater intrusion problem
}

\author{
Jiansong Zhang*, Yuezhi Zhang, Zhaohui Liu \\ Department of Applied Mathematics, China University of Petroleum, Qingdao 266580, China.
}

Communicated by S. H. Wu

\begin{abstract}
A new characteristic mixed finite element method is developed for solving saltwater intrusion problem. In this algorithm, the splitting mixed finite element (SMFE) method is applied for solving the parabolic-type water head equation, and the mass-conservative characteristic (MCC) finite element method is applied for solving the convection-diffusion type concentration equation. The application of the splitting mixed element method results in a symmetric positive definite coefficient matrix of the mixed element system and separating the flux equation from the water head equation. While the mass-conservative characteristic finite element method does well in handling convection-dominant diffusion problem and keeps mass balance. The convergence of this method is considered and the optimal L ${ }^{2}$-norm error estimate is also derived. (C) 2017 All rights reserved.
\end{abstract}

Keywords: Method of characteristics, mass-conservative, splitting mixed finite element, error estimate, saltwater intrusion problem.

2010 MSC: 65M12, 65M15, 65M25, 65M60.

\section{Introduction}

In this paper, we will consider the following coupled system composed of the water head equation and the concentration $\left(\mathrm{of} \mathrm{Cl}^{-}\right.$) equation as a mathematical model of seawater intrusion problems (see $[2,19])$ :

$$
\begin{aligned}
& \text { (a) } \mathrm{S}_{\mathrm{s}} \frac{\partial \mathrm{H}}{\partial \mathrm{t}}-\nabla \cdot\left(\widetilde{\kappa}\left(\nabla \mathrm{H}-\eta \mathrm{ce} \mathbf{e}_{3}\right)\right)=-\phi \eta \frac{\partial \mathrm{c}}{\partial \mathrm{t}}+\frac{\rho}{\rho_{0}} \mathrm{q}, \\
& \text { (b) } \phi \frac{\rho_{0}}{\rho} \frac{\partial \mathrm{c}}{\partial \mathrm{t}}+\nabla \cdot(\mathbf{u c}-\phi \mathrm{D} \nabla \mathrm{c})=\widetilde{\mathrm{c}} \mathrm{q} \\
& x \in \Omega, 0<\mathrm{t} \leqslant \mathrm{T}
\end{aligned}
$$

with the initial-boundary conditions:

$$
\begin{aligned}
\mathbf{u} \cdot v & =0, \quad D \nabla c \cdot v=0, \quad \text { on } \partial \Omega, \\
H(x, 0) & =H^{0}(x), \quad c(x, 0)=c^{0}(x), \quad x \in \Omega,
\end{aligned}
$$

where $\Omega$ is a convex bounded domain in $R^{3}$ with the boundary $\partial \Omega, S_{s}$ is the specific retention, $H=\frac{p}{\rho_{0} g}-z$

\footnotetext{
${ }^{*}$ Corresponding author

Email addresses: jszhang@upc .edu.cn (Jiansong Zhang), zhangyuezhi2015@163.com (Yuezhi Zhang), liuzhaohui2016@163.com (Zhaohui Liu)

doi:10.22436/jnsa.010.11.44
}

Received 2016-12-27 
is water head function, $p$ stands for pressure, $\rho_{0}$ represents the density of reference water (fresh water), $\mathrm{g}$ is gravitational acceleration, $z$ is the height of water containing layer; $\rho$ is density dependent only on the concentration of salt $c$, Hugakorn's linearization $\rho=\rho_{0}\left(1+\frac{c}{c_{s}}\right)$ is adopted, $c_{s}$ is the concentration corresponding to the maximum density, and $\varepsilon$ is the density difference ratio $\epsilon=\frac{\rho_{s}-\rho_{0}}{\rho_{0}} . \widetilde{\kappa}=\frac{\rho g}{\mu} \kappa, \mu$ is viscosity of the fluid, $\mathrm{k}$ is the permeability; $\eta=\frac{\epsilon}{c_{s}}$ is the density coupling coefficient; $\mathbf{e}_{3}=(0,0,1)^{\mathrm{T}}$; $\phi$ is the porosity; and $q$ is the source or sink term; c stands for the concentration of $\mathrm{Cl}^{-}, \widetilde{\mathrm{c}}$ is the salt concentration near the source well, D is the diffusion matrix, $\mathbf{u}=-\frac{\rho_{0}}{\rho} \kappa\left(\nabla \mathrm{H}-\eta \mathbf{c e}_{3}\right)$ is Darcy velocity; $v$ is the unit vector outer normal to $\partial \Omega$.

As we know, the seawater intrusion problem attracts great attentions recently since it causes great damages to industrial and agricultural production, which is urgent to be tackled. Thanks to the increasing interest, there are more and more literatures on the problem in recent years, see $[2,6,8,9,13,15]$. Many numerical methods were also established for simulating this problem, for example, finite difference method [22], finite element methods [19], upwind fractional-step finite difference methods [18, 20, 23], alternating-direction methods [21,24] and so on. However, these above proposed methods applied to the convection-dominated problems do not work well, and produce excessive numerical diffusion or nonphysical oscillation. Furthermore, solving the water head equation with the standard finite element method or finite difference method cannot directly obtain the approximate flux which appears in the concentration equation. The way to obtain the flux through differentiating the water head function will cause extra error and reduce the accuracy. To obtain more accurate approximation of the flux function, Lian and Rui gave a mixed finite element method combined with an inner penalty discontinuous Galerkin finite element method in [11]. But the technique of the classical mixed finite element method leads to some saddle point problem whose numerical solutions have been quite difficult because of losing positive definite properties. In [17, 25-30], Yang, Zhang etc. proposed a class of splitting mixed element methods, in which the mixed system is symmetric positive definite and the flux equation is separated from the original equation.

Moreover, the concentration equation is normally convection-dominated diffusion equation, and a more important property of the concentration equation is the mass balance. A variety of numerical techniques have been introduced to obtain better approximations, such as higher-order Godunov scheme [3], streamline diffusion method [10], least-squares mixed finite element method [16], and the EulerianLagrangian localized adjoint method (ELLAM) [4, 14]. Godunov schemes require that a CFL time-step constraint be imposed. Streamline diffusion method and least-squares mixed finite element method reduce the amount of diffusion but add a user-defined amount biased in the direction of the streamline. ELLAM conserves mass locally but it is difficult to evaluate the resulting integrals. The modified method of characteristic (MMOC) finite element method [7], has much smaller numerical diffusion, nonphysical oscillations, and time-truncation than those of standard methods, and can be used with a larger time step. Unfortunately, MMOC fails to maintain mass conservation. In [12], Rui and Tabata presented a new characteristic finite element method for convection-diffusion problems which not only keeps the advantages of the classical characteristic methods, but also preserves the global mass conservation.

In this paper, in order to solve saltwater intrusion problem, the splitting mixed element method is used to solve the water head equation of parabolic type and the mass-conservative characteristic finite element method is used to solve the concentration equation of convection-diffusion type. The splitting mixed element method has three advantages: 1 . it can obtain more accurate approximation value to the flux like classical mixed element methods; 2 . the coefficient matrix of the new mixed system is symmetric positive definite so that LBB condition required by classical mixed element methods is not necessary; 3 . the flux equation is separated from the water head equation, so that no coupled problem is solved. While the mass-conservative characteristic finite element method not only keeps the advantages of traditional MMOC and does well in handling convection-dominant diffusion problem, but also maintains the global mass conservation. Here, the convergence of this combined method is considered and an optimal L ${ }^{2}$-norm error estimate is also derived.

The structure of this paper is organized as follows: First, we present our method for saltwater intrusion 
problem and give the convergence theorem in Section 2. And then, we give some preliminaries, which will be used to prove our convergence theorem in Section 3. Next, we consider the error estimates for the concentration and the flux in Sections 4 and 5, respectively. Finally, we complete the proof of the convergence theorem in Section 6.

\section{Formulation of the method}

Throughout this paper, the notations of standard Sobolev spaces are adopted as in [1]. Let $(\cdot, \cdot)$ be the inner product in $\mathrm{L}^{2}(\Omega)$. Introduce the space $\mathrm{H}(\operatorname{div} ; \Omega)=\left\{\mathbf{v} \in\left[\mathrm{L}^{2}(\Omega)\right]^{3} ; \nabla \cdot \mathbf{v} \in \mathrm{L}^{2}(\Omega)\right\}, \mathcal{V}=\{\mathbf{v} \in$ $\mathrm{H}(\operatorname{div} ; \Omega) ; \mathbf{v} \cdot \boldsymbol{v}=0$ on $\partial \Omega\}$ and $\mathcal{W}=\mathrm{L}^{2}(\Omega)$. For convenience of analysis, we assume that the problem (1.1) is $\Omega$-periodic, i.e., all functions will be assumed to be spatially $\Omega$-periodic throughout the rest of this paper. This assumption is physically reasonable, because no-flow condition (1.2) is generally treated by reflection, and boundary effects are of less interest than interior flow patterns.

\subsection{The MCC for the concentration}

Define the differentiation along the characteristic curves of the transport $\frac{\phi}{\beta} \frac{\partial}{\partial t}+\mathbf{u} \cdot \nabla$,

$$
\psi(x, c, \mathbf{u}) \frac{\partial}{\partial \tau}=\frac{\phi}{\beta} \frac{\partial}{\partial t}+\mathbf{u} \cdot \nabla
$$

where $\beta=\rho(c) / \rho_{0}, \psi(x, c, \mathbf{u})=\sqrt{\phi^{2} / \beta^{2}(c)+|\mathbf{u}|^{2}}$. Note that the characteristic direction $\tau$ depends on $x$, the concentration $c$, and Darcy velocity $\mathbf{u}$, which vary in space and time. It follows easily that the concentration equation can be rewritten in the equivalent form

$$
\psi \frac{\partial \mathrm{c}}{\partial \tau}+\nabla \cdot \mathbf{u c}-\nabla \cdot(\phi \mathrm{D} \nabla \mathrm{c})=\widetilde{\mathbf{c}} \mathbf{q}
$$

Define the time partition for the concentration $0=: t_{0}^{c}<t_{1}^{c}<\cdots<t_{n}^{c}<\cdots<t_{N-1}^{c}<t_{N}^{c}:=T$, with $\Delta t_{\mathfrak{n}}^{c}=: t_{\mathfrak{n}}^{c}-t_{\mathfrak{n}-1}^{c}$. The characteristic derivative is approximated by

$$
\psi \frac{\partial c}{\partial \tau} \approx \psi \frac{c^{n}-c^{n-1} \circ X^{n}}{\sqrt{\left(X^{n}-x\right)^{2}+\left(t^{n}-t^{n-1}\right)^{2}}}=\frac{\phi}{\beta^{n-1}} \frac{c^{n}-c^{n-1} \circ X^{n}}{\Delta t_{n}^{c}},
$$

where

$$
c^{n-1} \circ X^{n}=c^{n-1}\left(X^{n}\right), \quad X^{n}(x)=x-\frac{\mathbf{u}^{n-1} \beta^{n-1}}{\phi} \Delta t_{n}^{c}, \quad \beta^{n-1}=\frac{\rho\left(c^{n-1}\right)}{\rho_{0}} .
$$

And then the traditional continuous-in-space characteristic procedure of the concentration equation (2.1) can be given by

$$
\frac{\phi}{\beta^{n-1}} \frac{c^{n}-c^{n-1} \circ X^{n}}{\Delta t_{n}^{c}}+\nabla \cdot \mathbf{u}^{n} c^{n}-\nabla \cdot\left(\phi D \nabla c^{n}\right)=\widetilde{c}^{n} q^{n} .
$$

As we know, this procedure can not keep the mass balance. In [12], a new mass-conservative characteristic algorithm was established. By use of the similar technique, the continuous-in-space MCC procedure of the concentration equation (2.1) can be written as follows

$$
\frac{1}{\triangle t_{n}^{c}}\left(\frac{\phi}{\beta^{n-1}} c^{n}-\left(\frac{\phi}{\beta^{n-1}} c^{n-1}\right) \circ X^{n} \delta^{n}\right)-\nabla \cdot\left(\phi D \nabla c^{n}\right)=\widetilde{c}^{n} q^{n},
$$

where $\delta^{n}$ is the Jacobian of the transformation $X^{n}$, that is,

$$
\delta^{n}=\operatorname{det}\left(\frac{\partial X^{n}}{\partial x}\right)=\operatorname{det}\left(\delta_{i j}-\Delta t_{n}^{c} \frac{\partial u_{i}^{n}}{\partial x_{j}}\right) .
$$

Let $\mathcal{T}_{h_{c}}$ be one quasi-regular finite element partition of the domain $\Omega$, such that the elements in the partitions have the diameters bounded by $h_{c}$. Let $\mathcal{M}_{h} \subset H^{1}(\Omega)$ be k-degree polynomial finite element spaces defined on the partition $\mathcal{T}_{h_{c}}$. The MCC finite element procedure can be given as follows: 
MCC Scheme. For given $\mathbf{u}_{h}^{n-1} \in \mathcal{V}_{h}$, seek by $c_{h}^{n} \in \mathcal{M}_{h}$ such that

$$
\frac{1}{\triangle \mathrm{t}_{\mathrm{n}}^{\mathrm{c}}}\left(\frac{\phi \mathrm{c}_{\mathrm{h}}^{\mathrm{n}}}{\beta_{\mathrm{h}}^{\mathrm{n}-1}}-\left(\frac{\phi \mathrm{c}_{\mathrm{h}}^{\mathrm{n}-1}}{\beta_{\mathrm{h}}^{\mathrm{n}-1}}\right) \circ \mathrm{X}^{\mathrm{n}} \delta^{\mathrm{n}}, z_{\mathrm{h}}\right)+\left(\phi D \nabla \mathrm{c}_{\mathrm{h}}^{\mathrm{n}}, \nabla z_{\mathrm{h}}\right)=\left(\widetilde{\mathrm{c}}_{\mathrm{h}}^{\mathrm{n}} \mathrm{q}^{\mathrm{n}}, z_{\mathrm{h}}\right), \quad \forall z_{\mathrm{h}} \in \mathcal{M}_{\mathrm{h}},
$$

where $\beta_{h}^{n-1}=\rho\left(c_{h}^{n-1}\right) / \rho_{0}, \quad X^{n}(x)=x-\triangle t_{n}^{c} \mathbf{u}_{h}^{n-1} \beta_{h}^{n-1} / \phi$.

As in [12], we will show the mass conservation of the MCC Scheme.

As we know that, the conversation of (1.1)-(b) requires

$$
\int_{\Omega} \frac{\phi}{\beta(c)} \frac{\partial c}{\partial t} d x=\int_{\Omega} \tilde{c} q d x .
$$

By integrating along time step, the discrete version of this equation can be written as

$$
\frac{1}{\Delta t_{n}^{c}} \int_{\Omega} \frac{\phi c_{h}^{n}}{\beta_{h}^{n-1}} d x=\frac{1}{\Delta t_{n}^{c}} \int_{\Omega} \frac{\phi c_{h}^{n-1}}{\beta_{h}^{n-1}} d x+\int_{\Omega} \tilde{c}_{h}^{n} q^{n} d x
$$

which can be regarded as a reasonable approximation.

In (2.2), we take $z_{\mathrm{h}}=1$, and we have

$$
\frac{1}{\Delta t_{n}^{c}} \int_{\Omega} \frac{\phi c_{h}^{n}}{\beta_{h}^{n-1}} d x=\frac{1}{\Delta t_{n}^{c}} \int_{\Omega}\left(\frac{\phi c_{h}^{n-1}}{\beta_{h}^{n-1}}\right) \circ X^{n} \delta^{n} d x+\int_{\Omega} \tilde{c}_{h}^{n} q^{n} d x
$$

Using the inverse transformation and $\Omega$-periodic boundary conditions, we know that

$$
\int_{\Omega}\left(\frac{\phi c_{h}^{n-1}}{\beta_{h}^{n-1}}\right) \circ X^{n} \delta^{n} d x=\int_{X^{n}(\Omega)}\left(\frac{\phi c_{h}^{n-1}}{\beta_{h}^{n-1}}\right)(y) \delta^{n}\left(\delta^{n}\right)^{-1} d y=\int_{\Omega} \frac{\phi c_{h}^{n-1}}{\beta_{h}^{n-1}} d x,
$$

which implies that MCC Scheme keeps the mass balance.

\subsection{The splitting mixed element scheme for water head and flux}

As we know, the water head equation is a parabolic type equation. Now we deal it with the splitting positive definite mixed finite element method. Define the flux $\sigma$ as follows:

$$
\boldsymbol{\sigma}=-\widetilde{\kappa}\left(\nabla H-\eta \mathbf{e}_{3}\right)=\frac{g \rho^{2}}{\rho_{0} \mu} \mathbf{u} .
$$

So we have $\mathbf{u}=a(c) \boldsymbol{\sigma}, a(c)=\rho_{0} \mu / g \rho^{2}$. A mixed weak form of the system (1.1)-(a) is given by:

$$
\begin{aligned}
& \text { (a) }\left(\frac{\partial \mathrm{H}}{\partial \mathrm{t}}, w\right)+(\mathrm{B} \nabla \cdot \boldsymbol{\sigma}, w)=(\mathrm{B} \beta \mathrm{q}, w)-\left(\mathrm{B} \phi \eta \frac{\partial \mathrm{c}}{\partial t}, w\right), \quad \forall w \in \mathcal{W}, \\
& \text { (b) }(\alpha(\mathrm{c}) \boldsymbol{\sigma}, \mathbf{v})-(\mathrm{H}, \nabla \cdot \mathbf{v})=-\left(\eta \mathbf{e}_{3}, \mathbf{v}\right), \quad \forall \mathbf{v} \in \mathcal{V},
\end{aligned}
$$

where $\alpha(c)=1 / \widetilde{K}$ and $B=1 / S_{s}$. From (2.3)-(b) we derive

$$
\left(\frac{\partial}{\partial t}(\alpha(c) \boldsymbol{\sigma}), \mathbf{v}\right)-\left(\frac{\partial H}{\partial t}, \nabla \cdot \mathbf{v}\right)=-\left(\eta \frac{\partial c}{\partial t} \mathbf{e}_{3}, \mathbf{v}\right) .
$$

Taking $w=\nabla \cdot \mathbf{v}$ in (2.3)-(a) and substituting it into (2.4), we get the mixed system

$$
\begin{aligned}
& \text { (a) }\left(\frac{\partial}{\partial t}(\alpha(\mathrm{c}) \boldsymbol{\sigma}), \mathbf{v}\right)+(\mathrm{B} \nabla \cdot \boldsymbol{\sigma}, \nabla \cdot \mathbf{v})=(\mathrm{B} \beta \mathrm{q}, \nabla \cdot \mathbf{v})-\left(\mathrm{B} \phi \eta \frac{\partial \mathrm{c}}{\partial \mathrm{t}}, \nabla \cdot \mathbf{v}\right)-\left(\eta \frac{\partial \mathrm{c}}{\partial \mathrm{t}} \mathbf{e}_{3}, \mathbf{v}\right), \quad \forall \mathbf{v} \in \mathcal{V}, \\
& \text { (b) }\left(\frac{\partial \mathrm{H}}{\partial \mathrm{t}}, w\right)=(\mathrm{B}[\beta \mathrm{q}-\nabla \cdot \boldsymbol{\sigma}], w)-\left(\mathrm{B} \phi \eta \frac{\partial \mathrm{c}}{\partial \mathrm{t}}, w\right), \quad \forall w \in \mathcal{W} .
\end{aligned}
$$

From system (2.5) we can see that the flux equation is separated from the water head equation and then the water head function $\mathrm{H}$, if required, can be obtained from (2.5)-(b) straightly.

We define a temporal partition on the time interval $[0, T]$ for the head water and flux grid by $0=$ : $\mathrm{t}_{0}^{\mathrm{H}}<\mathrm{t}_{1}^{\mathrm{H}}<\cdots<\mathrm{t}_{\mathrm{m}}^{\mathrm{H}}<\cdots<\mathrm{t}_{\mathrm{M}-1}^{\mathrm{H}}<\mathrm{t}_{M}^{\mathrm{H}}:=\mathrm{T}$, with $\Delta \mathrm{t}_{\mathrm{m}}^{\mathrm{H}}=: \mathrm{t}_{\mathrm{m}}^{\mathrm{H}}-\mathrm{t}_{\mathrm{m}-1}^{\mathrm{H}}$. Each water head step is also 
a concentration step, i.e., for each $m$ there exists $n$ such that $t_{n}^{c}=t_{m}^{H}$. Let $\mathcal{T}_{h_{\sigma}}, \mathcal{T}_{h_{H}}$ be two families of quasi-regular finite element partitions of the domain $\Omega$ which may be the same one or not, such that the elements in the partitions have the diameters bounded by $h_{\sigma}, h_{H}$, respectively. Let $\mathcal{V}_{h} \subset \mathcal{V}, \mathcal{W}_{h} \subset \mathcal{W}$ be $r$ and l-degree polynomial finite element spaces defined on the partitions $\mathcal{T}_{h_{\sigma}}$ and $\mathcal{T}_{h_{H}}$, respectively. A splitting mixed element procedure for water head and flux can be given as follows:

SMFE Scheme. Seek $\left(\sigma_{h}^{m}, H_{h}^{m}\right) \subset \mathcal{V}_{h} \times \mathcal{W}_{h}$ for given concentration $c_{h} \in \mathcal{M}_{h}$ such that

$$
\begin{aligned}
& \left(\frac{\alpha\left(c_{h}^{m}\right) \sigma_{h}^{m}-\alpha\left(c_{h}^{m-1}\right) \sigma_{h}^{m-1}}{\Delta t_{m}^{H}}, \mathbf{v}_{h}\right)+\left(B \nabla \cdot \sigma_{h}^{m}, \nabla \cdot \mathbf{v}_{h}\right) \\
& \quad=\left(B \beta_{h}^{n} q^{m}, \nabla \cdot \mathbf{v}_{h}\right)-\left(B \phi \eta \frac{c_{h}^{m}-c_{h}^{m-1}}{\triangle t_{m}^{H}}, \nabla \cdot \mathbf{v}_{h}\right)-\left(\eta \frac{c_{h}^{m}-c_{h}^{m-1}}{\triangle t_{m}^{H}} \mathbf{e}_{3}, \mathbf{v}_{h}\right), \quad \forall \mathbf{v}_{h} \in V_{h},
\end{aligned}
$$

(b) $\left(\frac{\mathrm{H}_{\mathrm{h}}^{\mathrm{m}}-\mathrm{H}_{\mathrm{h}}^{\mathrm{m}-1}}{\triangle \mathrm{t}_{\mathrm{m}}^{\mathrm{H}}}, w_{\mathrm{h}}\right)=\left(\mathrm{B}\left[\beta_{\mathrm{h}}^{\mathrm{m}} \mathrm{q}^{\mathrm{n}}-\nabla \cdot \boldsymbol{\sigma}_{\mathrm{h}}^{\mathrm{n}}\right], w_{\mathrm{h}}\right)-\left(\mathrm{B} \phi \eta \frac{\mathrm{c}_{\mathrm{h}}^{\mathrm{m}}-\mathrm{c}_{\mathrm{h}}^{\mathrm{m}-1}}{\Delta \mathrm{t}_{\mathrm{m}}^{\mathrm{H}}}, w_{\mathrm{h}}\right), \quad \forall w_{\mathrm{h}} \in \mathcal{W}_{\mathrm{h}}$.

\subsection{The combined approximation procedure}

Next, we will present a new mass-conservative characteristic splitting mixed finite element (MCCSMFE) method for solving saltwater intrusion problem. For convenience, we define a uniform time partition: $0=: t_{0}<t_{1}<\cdots<t_{n}=n \Delta t<\cdots<t_{N-1}<t_{N}:=T$, with $\triangle t=: t_{n}-t_{n-1}$. Combined the method of mass-conservative characteristics with the splitting mixed element procedure, a new numerical method can be established:

MCC-SMFE Algorithm. Give an initial approximation $\left(c_{h}^{0}, H_{h}^{0}, \sigma_{h}^{0}\right)=\left(P_{h} c^{0}, Q_{h} H^{0}, \Pi_{h} \sigma^{0}\right) \in \mathcal{M}_{h} \times \mathcal{W}_{h} \times$ $\mathcal{V}_{h}$, for $n=1,2, \ldots, N$, seek $\left(c_{h}^{n}, \sigma_{h}^{n}, H_{h}^{n}\right) \in \mathcal{M}_{h} \times \mathcal{V}_{h} \times \mathcal{W}_{h}$ such that

$$
\begin{aligned}
& \text { (a) } \frac{1}{\Delta t}\left(\frac{\phi c_{h}^{n}}{\beta_{h}^{n-1}}-\left(\frac{\phi c_{h}^{n-1}}{\beta_{h}^{n-1}}\right) \circ X^{n} \delta^{n}, z_{h}\right)+\left(\phi D \nabla c_{h}^{n}, \nabla z_{h}\right)=\left(\widetilde{c}_{h}^{n} q^{n}, z_{h}\right), \quad \forall z_{h} \in \mathcal{M}_{h}, \\
& \text { (b) }\left(\frac{\alpha\left(c_{h}^{n}\right) \sigma_{h}^{n}-\alpha\left(c_{h}^{n-1}\right) \sigma_{h}^{n-1}}{\Delta t}, \mathbf{v}_{h}\right)+\left(B \nabla \cdot \sigma_{h}^{n}, \nabla \cdot \mathbf{v}_{h}\right) \\
& \quad=\left(B \beta_{h}^{n} q^{n}, \nabla \cdot \mathbf{v}_{h}\right)-\left(B \phi \eta \frac{c_{h}^{n}-c_{h}^{n-1}}{\triangle t}, \nabla \cdot \mathbf{v}_{h}\right)-\left(\eta \frac{c_{h}^{n}-c_{h}^{n-1}}{\triangle t} \mathbf{e}_{3}, \mathbf{v}_{h}\right), \quad \forall \mathbf{v}_{h} \in \mathcal{V}_{h}, \\
& \text { (c) }\left(\frac{H_{h}^{n}-H_{h}^{n-1}}{\triangle t}, w_{h}\right)=\left(B\left[\beta_{h}^{n} q^{n}-\nabla \cdot \sigma_{h}^{n}\right], w_{h}\right)-\left(B \phi \eta \frac{c_{h}^{n}-c_{h}^{n-1}}{\Delta t}, w_{h}\right), \quad \forall w_{h} \in \mathcal{W}_{h},
\end{aligned}
$$

where the definitions of the projection operators $P_{h}, \Pi_{h}$, and $Q_{h}$, can be found in Section 3 .

For convenience of analysis, we assume that $K$ and $\varepsilon$ indicate a generic constant and a small positive constant independent of mesh parameters $h_{\sigma}, h_{H}, h_{c}$ and time increment $\Delta t$, which may be different at their occurrences. We assume that the diffusion matrix $D$ is independent on the concentration $c$ as in $[11,19]$, and make the following hypotheses

$$
\begin{aligned}
& 0<\phi_{*} \leqslant \phi \leqslant \phi^{*}, \quad 0<D_{*} \leqslant D \leqslant D^{*}, \quad 0<S_{*} \leqslant S_{s} \leqslant S^{*}, \\
& 0<\alpha_{*} \leqslant \alpha \leqslant \alpha^{*}, \quad 0<\beta_{*} \leqslant \beta \leqslant \beta^{*}, \quad 0<a_{*} \leqslant a \leqslant a^{*}, \\
& \left|\frac{\partial \alpha(c)}{\partial c}\right|+\left|\frac{\partial \beta(c)}{\partial c}\right|+\left|\frac{\partial a(c)}{\partial c}\right|+\left|\frac{\partial^{2} a(c)}{\partial c^{2}}\right| \leqslant K^{*} .
\end{aligned}
$$

Moreover, we also assume the regularities of the solution of (1.1)-(1.2) as follows:

$$
\begin{array}{rlrl}
c & \in \mathrm{L}^{\infty}\left(\mathrm{H}^{\mathrm{k}+1}\right) \cap \mathrm{L}^{2}\left(\mathrm{~W}_{\infty}^{1}\right), & & \frac{\partial c}{\partial \mathrm{t}} \in \mathrm{L}^{2}\left(\mathrm{H}^{\mathrm{k}+1}\right) \cap \mathrm{L}^{\infty}\left(\mathrm{L}^{\infty}\right), \\
\frac{\partial^{2} \mathrm{c}}{\partial \mathrm{t}^{2}} \in \mathrm{L}^{2}\left(\mathrm{~L}^{2}\right), & \mathrm{H} \in \mathrm{L}^{\infty}\left(\mathrm{H}^{\mathrm{l}+1}\right) \cap \mathrm{H}^{2}\left(\mathrm{~L}^{2}\right), & \\
\boldsymbol{\sigma} & \in \mathrm{L}^{\infty}\left(\mathrm{H}^{\mathrm{r}+1} \cap \mathrm{W}_{\infty}^{1}\right), & \frac{\partial \boldsymbol{\sigma}}{\partial \mathrm{t}} \in \mathrm{L}^{2}\left(\mathrm{H}^{\mathrm{r}+1}\right) \cap \mathrm{L}^{\infty}\left(\mathrm{L}^{\infty}\right), & \frac{\partial^{2} \boldsymbol{\sigma}}{\partial \mathrm{t}^{2}} \in \mathrm{L}^{2}\left(\mathrm{~L}^{2}\right) .
\end{array}
$$


For MCC-SMFE Algorithm, we have the following main result.

Theorem 2.1. Assume that the hypotheses (2.6) hold and the solution of system (1.1)-(1.2) has the regular properties (2.7). If the mesh parameters $h_{c}, h_{\sigma}$, and $\triangle t$ satisfy the relations

$$
\triangle \mathrm{t}=\mathrm{o}\left(\mathrm{h}_{\mathrm{c}}^{\frac{3}{2}}\right)=\mathrm{o}\left(\mathrm{h}_{\mathrm{\sigma}}^{\frac{3}{2}}\right)
$$

then there hold the priori error estimates

$$
\begin{aligned}
& \text { (a) } \max _{n}\left\|c^{n}-c_{h}^{n}\right\|_{L^{2}}+\max _{n}\left\|\sigma^{n}-\sigma^{n}\right\|_{L^{2}} \leqslant K\left\{h_{c}^{k+1}+h_{\sigma}^{r+1}+h_{\sigma}^{r_{1}+1}+\Delta t\right\}, \\
& \text { (b) } \max _{n}\left\|H^{n}-H_{h}^{n}\right\|_{L^{2}} \leqslant K\left\{h_{c}^{k+1}+h_{\sigma}^{r+1}+h_{\sigma}^{r_{1}}+h_{H}^{l+1}+\triangle t\right\},
\end{aligned}
$$

where $\mathrm{K}$ is a constant independent of the parameters $\Delta \mathrm{t}, \mathrm{h}_{\mathrm{H}}, \mathrm{h}_{\sigma}, \mathrm{h}_{\mathrm{c}} ; \mathrm{r}, \mathrm{k}>0, \mathrm{l} \geqslant 0$ denote some integers, $\mathrm{r}_{1}=\mathrm{r}$ in cases of BDDM, BDM, and BDFM elements, or $r_{1}=r+1$ in cases of RT and Nedelec elements.

\section{Some preliminaries}

We assume that finite element spaces $\mathcal{V}_{\mathrm{h}}$ and $\mathcal{W}_{\mathrm{h}}$ have the approximate properties (see [5]) that there exist some integers $r, r_{1}>0$ and $l \geqslant 0$, such that, for $1 \leqslant q \leqslant \infty$,

$$
\begin{aligned}
\inf _{\mathbf{v}_{\mathrm{h}} \in \mathcal{V}_{\mathrm{h}}}\left\|\mathbf{v}-\mathbf{v}_{\mathrm{h}}\right\|_{[\mathrm{q}} \leqslant \mathrm{K}_{1} \mathrm{~h}_{\sigma}^{\mathrm{r}+1}\|\mathbf{v}\|_{W^{\mathrm{r}+1, \mathrm{q}},}, & \forall \mathbf{v} \in \mathrm{H}(\operatorname{div} ; \Omega) \cap \mathrm{W}^{\mathrm{r}+1, \mathrm{q}}(\Omega), \\
\inf _{\mathbf{v}_{\mathrm{h}} \in \mathcal{V}_{\mathrm{h}}}\left\|\nabla \cdot\left(\mathbf{v}-\mathbf{v}_{\mathrm{h}}\right)\right\|_{\mathrm{L}_{\mathrm{q}}} \leqslant \mathrm{K}_{1} \mathrm{~h}_{\sigma}^{\mathrm{r}_{1}}\|\nabla \cdot \mathbf{v}\|_{\mathrm{W}^{\mathrm{r}_{1}, \mathrm{q}},}, & \forall \mathbf{v} \in \mathrm{H}(\operatorname{div} ; \Omega) \cap \mathrm{W}^{\mathrm{r}_{1}+1, \mathrm{q}}(\Omega), \\
\inf _{\mathcal{W}_{\mathrm{h}} \in \mathcal{W}_{\mathrm{h}}}\left\|w-\mathcal{w}_{\mathrm{h}}\right\|_{\mathrm{L}^{\mathrm{q}}} \leqslant \mathrm{K}_{1} \mathrm{~h}_{\mathrm{H}}^{\mathrm{l}+1}\|w\|_{W^{\mathrm{l}+1, \mathrm{q}}}, & \forall w \in \mathrm{L}^{2}(\Omega) \cap \mathrm{W}^{\mathrm{l}+1, \mathrm{q}}(\Omega) .
\end{aligned}
$$

It is well-known that, in any one of the classical mixed finite element spaces, there exists an operator $\Pi_{\mathrm{h}}$ from $\mathcal{V}$ onto $\mathcal{V}_{\mathrm{h}}$, see [5], such that, for any $1 \leqslant \mathrm{q} \leqslant+\infty$,

$$
\begin{aligned}
& \text { (a) }\left(\nabla \cdot\left(\mathbf{v}-\Pi_{\mathrm{h}} \mathbf{v}\right), \nabla \cdot \mathbf{v}_{\mathrm{h}}\right)=0, \quad \forall \mathbf{v}_{\mathrm{h}} \in \mathcal{V}_{\mathrm{h}}, \\
& \text { (b) }\left\|\mathbf{v}-\Pi_{\mathrm{h}} \mathbf{v}\right\|_{\mathrm{L}^{\mathrm{q}}} \leqslant \mathrm{Kh}_{\sigma}^{\mathrm{r}+1}\|\mathbf{v}\|_{W^{\mathrm{r}+1, \mathrm{q}}}, \\
& \text { (c) }\left\|\nabla \cdot\left(\mathbf{v}-\Pi_{\mathrm{h}} \mathbf{v}\right)\right\|_{\mathrm{L} q} \leqslant \mathrm{Kh}_{\sigma}^{\mathrm{r}_{1}}\|\nabla \cdot \mathbf{v}\|_{W^{\mathrm{r}_{1}, \mathrm{q}}}
\end{aligned}
$$

And we define a projection operator $\mathrm{P}_{h}$ from $\mathrm{H}^{1}(\Omega)$ onto $\mathcal{M}_{h}$ such that, for all $z_{h} \in \mathcal{M}_{h}$ and $c \in H^{1}$,

$$
\left(\phi \mathrm{D} \nabla \mathrm{c}, \nabla z_{\mathrm{h}}\right)=\left(\phi \mathrm{D} \nabla \mathrm{P}_{\mathrm{h}} \mathrm{c}, \nabla z_{\mathrm{h}}\right) .
$$

By use of the inverse property and approximate properties of the finite element space $\mathcal{M}_{h}$ (see [5]), the following error bounds were given: for some integer $k>0$,

$$
\begin{aligned}
& \text { (a) }\left\|\mathrm{c}-\mathrm{P}_{\mathrm{h}} \mathrm{c}\right\|_{\mathrm{L}^{2}}+\mathrm{h}_{\mathrm{c}}\left\|\nabla\left(\mathrm{c}-\mathrm{P}_{\mathrm{h}} \mathrm{c}\right)\right\|_{\mathrm{L}^{2}} \leqslant \mathrm{Kh}_{\mathrm{c}}^{\mathrm{k}+1}\|\mathrm{c}\|_{\mathrm{H}^{\mathrm{k}+1}}, \\
& \text { (b) }\left\|\nabla \mathrm{P}_{\mathrm{h}} \mathrm{c}\right\|_{\mathrm{L}^{\infty}} \leqslant \mathrm{K}(\mathrm{c})<+\infty \\
& \text { (c) }\left\|\frac{\partial\left(\mathrm{c}-\mathrm{P}_{\mathrm{h}} \mathrm{c}\right)}{\partial \mathrm{t}}\right\|_{\mathrm{L}^{2}} \leqslant \mathrm{Kh}_{\mathrm{c}}^{\mathrm{k}+1}\left\{\|\mathrm{c}\|_{\mathrm{H}^{\mathrm{k}+1}}+\left\|\frac{\partial \mathrm{c}}{\partial \mathrm{t}}\right\|_{\mathrm{H}^{\mathrm{k}+1}}\right\} .
\end{aligned}
$$

Meanwhile, we also introduce the $L^{2}$ projection operator $Q_{h}$ from $L^{2}(\Omega)$ onto $\mathcal{W}_{h}$ such that

$$
\left(\mathrm{H}-\mathrm{Q}_{\mathrm{h}} \mathrm{H}, w_{\mathrm{h}}\right)=0, \quad \forall w_{\mathrm{h}} \in \mathcal{W}_{\mathrm{h}} .
$$

It is well-known that the a priori error estimate: for $l \geqslant 0$

$$
\left\|\mathrm{H}-\mathrm{Q}_{\mathrm{h}} \mathrm{H}\right\|_{\mathrm{L}^{2}} \leqslant \mathrm{Kh}_{\mathrm{H}}^{\mathrm{l}+1}\|\mathrm{H}\|_{\mathrm{H}^{\mathrm{l}+1}}, \quad \forall w \in \mathrm{H}^{\mathrm{l}+1}(\Omega)
$$

holds.

Next, we will give a lemma which is important to prove our theoretical result in the following sections. 
Lemma 3.1 ([17]). Assume that the finite element space $\mathcal{V}_{\mathrm{h}}$ is any one of the classical mixed finite element spaces defined in [5]. The super-approximation,

$$
\begin{aligned}
& \left(\varphi \nabla \cdot\left(\mathbf{v}-\Pi_{\mathrm{h}} \mathbf{v}\right), \nabla \cdot \mathbf{v}_{\mathrm{h}}\right) \\
& \quad \leqslant \mathrm{Kh}_{\sigma}\left\|\nabla \cdot \mathbf{v}_{\mathrm{h}}\right\|_{\mathrm{L}^{2}} \min \left(\| \varphi \| _ { \mathrm { H } ^ { 1 } } \| \nabla \cdot ( \mathbf { v } - \Pi _ { \mathrm { h } } \mathbf { v } ) \| _ { \mathrm { L } ^ { \infty } , \operatorname { m i n } } \left(\|\varphi\|_{\left.\left.\mathrm{W}^{1, \infty}, \mathrm{h}_{\sigma^{2}}^{-\frac{3}{2}}\right)\left\|\nabla \cdot\left(\mathbf{v}-\Pi_{\mathrm{h}} \mathbf{v}\right)\right\|_{\mathrm{L}^{2}}\right)}\right.\right.
\end{aligned}
$$

holds, for each function $\varphi \in \mathrm{W}^{1, \infty}, \mathbf{v} \in \mathcal{V}$ and $\mathbf{v}_{\mathrm{h}} \in \mathcal{V}_{\mathrm{h}}$.

\section{Error estimate for the concentration}

In order to derive the error estimate for the concentration, we make an induction hypothesis as follows:

$$
\max _{n}\left\|\mathbf{u}_{h}^{n}\right\|_{\infty} \leqslant k h_{\sigma}^{-\frac{1}{2}}\left[\frac{h_{\sigma}^{\frac{3}{2}}}{\Delta t}\right]^{\frac{1}{2}}
$$

Obviously, from MCC-SMFE Algorithm, we know that the induction hypothesis (4.1) holds when $n=0$. Moreover, we assume that (4.1) holds until $n=N-1$. Set $\xi_{c}^{n}=c_{h}^{n}-P_{h} c^{n}$ and $\zeta_{c}^{n}=c^{n}-P_{h} c^{n}$. By the definition of projection operator $P_{h}$, we have the error residual equation:

$$
\begin{aligned}
& \left(\frac{\phi}{\beta_{h}^{n-1}} \frac{\xi_{c}^{n}-\xi_{c}^{n-1}}{\Delta t}, z_{h}\right)+\left(\phi D \nabla \xi_{c}^{n}, \nabla z_{h}\right) \\
& =\left(\frac{\phi}{\beta_{h}^{n-1}} \frac{\partial c}{\partial t}+\mathbf{u}_{h}^{n-1} \cdot \nabla c^{n}+\nabla \cdot \mathbf{u}_{h}^{n-1} c^{n}-\frac{1}{\Delta t}\left(\frac{\phi}{\beta_{h}^{n-1}} c^{n}-\left(\frac{\phi}{\beta_{h}^{n-1}} c^{n-1}\right) \circ X^{n} \delta^{n}\right), z_{h}\right) \\
& +\left(\frac{\phi\left(\beta_{h}^{n}-\beta^{n-1}\right)}{\beta_{h}^{n-1} \beta^{n-1}} \frac{\partial c}{\partial t}, z_{h}\right)+\left(\frac{1}{\triangle t}\left(\left(\frac{\phi}{\beta_{h}^{n-1}} \xi_{c}^{n-1}\right) \circ X^{n} \delta^{n}-\frac{\phi}{\beta_{h}^{n-1}} \xi_{c}^{n-1}\right), z_{h}\right) \\
& +\left(\frac{\phi}{\beta_{h}^{n-1} \triangle t}\left(\zeta_{c}^{n}-\zeta_{c}^{n-1}\right), z_{h}\right)+\left(\frac{1}{\triangle t}\left(\frac{\phi}{\beta_{h}^{n-1}} \zeta_{c}^{n-1}-\left(\frac{\phi}{\beta_{h}^{n-1}} \zeta_{c}^{n-1}\right) \circ X^{n} \delta^{n}\right), z_{h}\right) \\
& +\left(\nabla \cdot\left(\left(\mathbf{u}^{n}-\mathbf{u}_{h}^{n-1}\right) c^{n}\right), d_{t} \xi_{c}^{n}\right)+\left(q^{n} \widetilde{\xi}_{c}^{n}, z_{h}\right)-\left(q^{n} \widetilde{\zeta}_{c}^{n}, z_{h}\right) .
\end{aligned}
$$

We have the following approximate result.

Lemma 4.1. Assume that $\beta, \beta^{\prime}, a, a^{\prime}, \phi$, and $\phi^{\prime}$ are bounded, then there exists an estimate

$$
\begin{aligned}
& \sum_{n=1}^{N}\left\|d_{t} \xi_{c}^{n}\right\|_{L^{2}}^{2} \Delta t+\left\|\nabla \xi_{c}^{N}\right\|_{L^{2}}^{2} \\
& \quad \leqslant K\left\{\sum_{n=1}^{N-1}\left[\left\|\xi_{c}^{n}\right\|_{L^{2}}^{2}+\left\|\xi_{\sigma}^{n}\right\|_{L^{2}}^{2}+\left\|\nabla \xi_{c}^{n}\right\|_{L^{2}}^{2}\right] \Delta t+h_{c}^{2 k+2}+h_{\sigma}^{2 r+2}+(\Delta t)^{2}\right\} .
\end{aligned}
$$

Proof. Denote $d_{t} f^{n}:=\left(f^{n}-f^{n-1}\right) / \Delta t$. Taking $z_{h}=d_{t} \xi_{c}^{n}$ in (4.2), then we have

$$
\begin{aligned}
\left(\frac{\phi}{\beta_{h}^{n-1}}\right. & \left.\frac{\xi_{c}^{n}-\xi_{c}^{n-1}}{\triangle t}, d_{t} \xi_{c}^{n}\right)+\left(\phi D \nabla \xi_{c}^{n}, \nabla d_{t} \xi_{c}^{n}\right) \\
= & \left(\frac{\phi}{\beta_{h}^{n-1}} \frac{\partial c}{\partial t}+\mathbf{u}_{h}^{n-1} \cdot \nabla c^{n}+\nabla \cdot \mathbf{u}_{h}^{n-1} c^{n}-\frac{1}{\triangle t}\left(\frac{\phi}{\beta_{h}^{n-1}} c^{n}-\left(\frac{\phi}{\beta_{h}^{n-1}} c^{n-1}\right) \circ X^{n} \delta^{n}\right), d_{t} \xi_{c}^{n}\right) \\
& +\left(\frac{\phi\left(\beta_{h}^{n}-\beta^{n-1}\right)}{\beta_{h}^{n-1} \beta^{n-1}} \frac{\partial c}{\partial t}, d_{t} \xi_{c}^{n}\right)+\left(\frac{1}{\triangle t}\left(\left(\frac{\phi}{\beta_{h}^{n-1}} \xi_{c}^{n-1}\right) \circ X^{n} \delta^{n}-\frac{\phi}{\beta_{h}^{n-1}} \xi_{c}^{n-1}\right), d_{t} \xi_{c}^{n}\right) \\
& +\left(\frac{\phi}{\beta_{h}^{n-1} \triangle t}\left(\zeta_{c}^{n}-\zeta_{c}^{n-1}\right), d_{t} \xi_{c}^{n}\right)+\left(\frac{1}{\triangle t}\left(\frac{\phi}{\beta_{h}^{n-1}} \zeta_{c}^{n-1}-\left(\frac{\phi}{\beta_{h}^{n-1}} \zeta_{c}^{n-1}\right) \circ X^{n} \delta^{n}\right), d_{t} \xi_{c}^{n}\right) \\
& +\left(\nabla \cdot\left(\left(\mathbf{u}^{n}-\mathbf{u}_{h}^{n-1}\right) c^{n}\right), d_{t} \xi_{c}^{n}\right)+\left(q^{n} \widetilde{\xi}_{c}^{n}, d_{t} \xi_{c}^{n}\right)-\left(q^{n} \widetilde{\zeta}_{c}^{n}, z_{h}\right) .
\end{aligned}
$$


The left-hand side of (4.4) is bigger than the quantity

$$
\frac{\alpha_{*}}{\beta^{*}}\left\|d_{t} \xi_{c}^{n}\right\|_{L^{2}}^{2}+\frac{1}{2} d_{t}\left((\phi D) \nabla \xi_{c}^{n}, \nabla \xi_{c}^{n}\right)-\frac{1}{2}\left(d_{t}(\phi D) \nabla \xi_{c}^{n-1}, \nabla \xi_{c}^{n-1}\right),
$$

and

$$
\frac{1}{2}\left(\mathrm{~d}_{\mathrm{t}}(\phi \mathrm{D}) \nabla \xi_{\mathrm{c}}^{\mathrm{n}-1}, \nabla \xi_{\mathrm{c}}^{\mathrm{n}-1}\right) \leqslant \mathrm{K}\left(1+\left\|\mathrm{d}_{\mathrm{t}} \beta^{\mathrm{n}-1}\right\|_{0, \infty}^{2}\right)\left\|\nabla \xi_{\mathrm{c}}^{\mathrm{n}-1}\right\|_{\mathrm{L}^{2}}^{2} .
$$

Now we multiply the relation (4.4) by $\Delta t$, sum over $1 \leqslant n \leqslant N$ and denote the resulting right-hand terms by $T_{1}, T_{2}, \ldots, T_{8}$. We turn to analyze these terms one by one.

$$
\begin{aligned}
\mathrm{T}_{1}= & \sum_{n=1}^{N}\left(\frac{\phi}{\beta_{h}^{n-1}} \frac{\partial c}{\partial t}+\mathbf{u}_{h}^{n-1} \cdot \nabla c^{n}+\nabla \cdot \mathbf{u}_{h}^{n-1} c^{n}-\frac{1}{\Delta t}\left(\frac{\phi}{\beta_{h}^{n-1}} c^{n}-\left(\frac{\phi}{\beta_{h}^{n-1}} c^{n-1}\right) \circ X^{n} \delta^{n}\right), d_{t} \xi_{c}^{n}\right) \Delta t \\
= & \sum_{n=1}^{N}\left(\frac{\phi}{\beta_{h}^{n-1}} \frac{\partial c}{\partial t}+\mathbf{u}_{h}^{n-1} \cdot \nabla c^{n}-\frac{\phi}{\beta_{h}^{n-1}} \frac{c^{n}-c^{n-1} \circ X^{n}}{\Delta t}, d_{t} \xi_{c}^{n}\right) \triangle t \\
& +\sum_{n=1}^{N}\left(\nabla \cdot \mathbf{u}_{h}^{n-1} c^{n}+\frac{1}{\triangle t}\left(\left(\frac{\phi}{\beta_{h}^{n-1}} c^{n-1}\right) \circ X^{n} \delta^{n}-\left(\frac{\phi}{\beta_{h}^{n-1}} c^{n-1}\right) \circ X^{n}\right), d_{t} \xi_{c}^{n}\right) \triangle t \\
& +\sum_{n=1}^{N}\left(\frac{1}{\triangle t}\left(\left(\frac{\phi}{\beta_{h}^{n-1}} c^{n-1}\right) \circ X^{n}-\frac{\phi}{\beta_{h}^{n-1}} c^{n-1} \circ X^{n}\right), d_{t} \xi_{c}^{n}\right) \triangle t \\
= & T_{11}+T_{12}+T_{13} .
\end{aligned}
$$

For $\mathrm{T}_{11}$ we have the estimate

$$
\mathrm{T}_{11} \leqslant \mathrm{~K}\left\|\frac{\partial^{2} \mathrm{c}}{\partial \mathrm{t}^{2}}\right\|_{\mathrm{L}^{2}\left((0, \mathrm{~T}] ; \mathrm{L}^{2}(\Omega)\right)}^{2} \Delta \mathrm{t}^{2}+\varepsilon \sum_{n=1}^{\mathrm{N}}\left\|\mathrm{d}_{\mathrm{t}} \xi_{\mathrm{c}}^{n}\right\|_{\mathrm{L}^{2}}^{2} \Delta \mathrm{t} .
$$

Based on the definite of $\delta^{n}$ and Taylor expansion, $\mathrm{T}_{12}$ can be decomposed as

$$
\begin{aligned}
& \mathrm{T}_{12}=\sum_{n=1}^{N}\left(\nabla \cdot \mathbf{u}_{h}^{n-1} c^{n}+\frac{1}{\triangle t}\left(\left(\frac{\phi}{\beta_{h}^{n-1}} c^{n-1}\right) \circ X^{n} \delta^{n}-\left(\frac{\phi}{\beta_{h}^{n-1}} c^{n-1}\right) \circ X^{n}\right), d_{t} \xi^{n}\right) \triangle t \\
& =\sum_{n=1}^{N}\left(\nabla \cdot \mathbf{u}_{h}^{n-1}\left(c^{n}-c^{n-1} \circ X^{n}\right), d_{t} \xi_{c}^{n}\right) \Delta t \\
& +\sum_{n=1}^{N}\left(\nabla \cdot \mathbf{u}_{h}^{n-1} c^{n-1} \circ X^{n}+\left(\frac{\phi}{\beta_{h}^{n-1}} c^{n-1}\right) \circ X^{n} \frac{\delta^{n}-1}{\Delta t}, d_{t} \xi_{c}^{n}\right) \Delta t \\
& \leqslant \mathrm{~K} \triangle \mathrm{t}^{2}\|\mathrm{c}\|_{\mathrm{H}^{1}\left(\mathrm{~L}^{2}\right) \cap \mathrm{C}^{0}\left(\mathrm{H}^{2}\right)}^{2}+\varepsilon \sum_{\mathrm{n}=1}^{\mathrm{N}}\left\|\mathrm{d}_{\mathrm{t}} \xi_{\mathrm{c}}^{n}\right\|_{\mathrm{L}^{2}}^{2} \triangle \mathrm{t}
\end{aligned}
$$

where we have used the induction hypothesis (4.1). Substituting (4.6) and (4.7) into (4.5), we have

$$
\mathrm{T}_{1} \leqslant \mathrm{~K}\left(\|\mathrm{c}\|_{\mathrm{H}^{1}\left(\mathrm{~L}^{2}\right)}^{2}+\|\mathrm{c}\|_{\mathrm{C}^{0}\left(\mathrm{H}^{2}\right)}^{2}+\left\|\frac{\partial^{2} \mathrm{c}}{\partial \mathrm{t}^{2}}\right\|_{\mathrm{L}^{2}\left((0, \mathrm{~T}] ; \mathrm{L}^{2}(\Omega)\right)}^{2}\right) \triangle \mathrm{t}^{2}+\varepsilon \sum_{n=1}^{\mathrm{N}}\left\|\mathrm{d}_{\mathrm{t}} \xi_{\mathrm{c}}^{n}\right\|_{\mathrm{L}^{2}}^{2} \triangle \mathrm{t} .
$$

For $T_{2}, T_{7}$, we have

$$
\mathrm{T}_{2}+\mathrm{T}_{7} \leqslant \mathrm{~K} \sum_{n=1}^{\mathrm{N}}\left\{\left\|\xi_{\mathrm{c}}^{\mathrm{n}}\right\|_{\mathrm{L}^{2}}^{2}+\Delta \mathrm{t}\left\|\frac{\partial \mathrm{c}}{\partial \mathrm{t}}\right\|_{\mathrm{L}^{2}\left((0, \mathrm{~T}] ; \mathrm{L}^{2}(\Omega)\right)}^{2}\right\} \triangle \mathrm{t}+\varepsilon \sum_{n=1}^{\mathrm{N}}\left\|\mathrm{d}_{\mathrm{t}} \xi_{\mathrm{c}}^{n}\right\|_{\mathrm{L}^{2}}^{2} \Delta \mathrm{t}+\mathrm{K} \mathrm{h}_{\mathrm{c}}^{2 \mathrm{k}+2} .
$$


For $T_{3}$, using the fact that $\xi_{c}^{0}=0$, we have

$$
\begin{aligned}
T_{3}= & \sum_{n=1}^{N}\left(\frac{\left(\frac{\phi}{\beta_{h}^{n-1}} \xi_{c}^{n-1}\right) \circ X^{n} \delta^{n}-\frac{\phi}{\beta_{h}^{n-1}} \xi_{c}^{n-1}}{\Delta t}, d_{t} \xi_{c}^{n}\right) \triangle t \\
= & \frac{1}{\Delta t} \sum_{n=1}^{N}\left(\left(\frac{\phi}{\beta_{h}^{n-1}} \xi_{c}^{n-1}\right) \circ X^{n} \delta^{n}-\frac{\phi}{\beta_{h}^{n-1}} \xi_{c}^{n-1}, \xi_{c}^{n}\right) \\
& -\frac{1}{\triangle t} \sum_{n=1}^{N}\left(\left(\frac{\phi}{\beta_{h}^{n-1}} \xi_{c}^{n-1}\right) \circ X^{n} \delta^{n}-\frac{\phi}{\beta_{h}^{n-1}} \xi_{c}^{n-1}, \xi_{c}^{n-1}\right) \\
= & -\sum_{n=2}^{N}\left(d_{t}\left(\left(\frac{\phi}{\beta_{h}^{n-1}} \xi_{c}^{n-1}\right) \circ X^{n} \delta^{n}-\frac{\phi}{\beta_{h}^{n-1}} \xi_{c}^{n-1}\right), \xi_{c}^{n-1}\right) \\
& +\left(\frac{\left(\phi \xi_{c}^{N-1} / \beta_{h}^{N-1}\right) \circ X^{N} \delta^{N}-\phi \xi_{c}^{N-1} / \beta_{h}^{N-1}}{\triangle t}, \xi_{c}^{N}\right)=-T_{31}+T_{32} .
\end{aligned}
$$

Note that

$$
\begin{aligned}
& \mathrm{T}_{31}=\sum_{n=2}^{N}\left(d_{t}\left(\left(\frac{\phi}{\beta_{h}^{n-1}} \xi_{c}^{n-1}\right) \circ X^{n} \delta^{n}-\frac{\phi}{\beta_{h}^{n-1}} \xi_{c}^{n-1}\right), \xi_{c}^{n-1}\right) \\
& =\sum_{n=2}^{N}\left(d_{t}\left(\left(\frac{\phi}{\beta_{h}^{n-1}} \xi_{c}^{n-1}\right) \circ X^{n} \delta^{n}\right), \frac{\xi_{c}^{n-1}-\xi_{c}^{n-1} \circ X^{n}}{\triangle t}\right) \triangle t \\
& +\sum_{n=2}^{N}\left(\left(\frac{\phi}{\beta_{h}^{n-1}} \xi_{c}^{n-1}\right) \circ X^{n} \delta^{n}-\left(\frac{\phi}{\beta_{h}^{n-2}} \xi_{c}^{n-2}\right) \circ X^{n-1} \delta^{n-1}, \frac{\xi_{c}^{n-1} \circ X^{n}}{\triangle t}\right) \\
& -\sum_{n=2}^{N}\left(\frac{\phi}{\beta_{h}^{n-1}} \xi_{c}^{n-1}-\frac{\phi}{\beta_{h}^{n-2}} \xi_{c}^{n-2}, \frac{\xi_{c}^{n-1}}{\triangle t}\right) \\
& =\sum_{n=2}^{N}\left(d_{t}\left(\left(\frac{\phi}{\beta_{h}^{n-1}} \xi_{c}^{n-1}\right) \circ X^{n} \delta^{n}\right), \frac{\xi_{c}^{n-1}-\xi_{c}^{n-1} \circ X^{n}}{\triangle t}\right) \triangle t \\
& +\sum_{n=2}^{N}\left(\left(\frac{\phi}{\beta_{h}^{n-2}} \xi_{c}^{n-2}\right) \circ X^{n-1} \delta^{n-1}, \frac{\xi_{c}^{n-1} \circ X^{n-1}-\xi_{c}^{n-1} \circ X^{n}}{\Delta t}\right) \triangle t=T_{311}+T_{312}
\end{aligned}
$$

where we have used the fact in the third equation that

$$
\begin{aligned}
\left(\left(\frac{\phi}{\beta_{h}^{n-1}} \xi_{c}^{n-1}\right) \circ X^{n} \delta^{n}, \frac{\xi_{c}^{n-1} \circ X^{n}}{\Delta t}\right) & =\left(\frac{\phi}{\beta_{h}^{n-1}} \xi_{c}^{n-1}, \frac{\xi_{c}^{n-1}}{\Delta t}\right), \\
\left(\left(\frac{\phi}{\beta_{h}^{n-2}} \xi_{c}^{n-2}\right) \circ X^{n-1} \delta^{n-1}, \frac{\xi_{c}^{n-1} \circ X^{n-1}}{\triangle t}\right) & =\left(\frac{\phi}{\beta_{h}^{n-2}} \xi_{c}^{n-2}, \frac{\xi_{c}^{n-1}}{\Delta t}\right) .
\end{aligned}
$$

For fixed $\bar{z}$, considering the transformation

$$
y=f_{\bar{z}}(x)=x-\frac{u^{n-1} \beta^{n-1}}{\phi} \Delta t \bar{z},
$$

we can find that $\operatorname{det}\left(\mathrm{Df}_{\bar{z}}\right)=\mathrm{O}\left(\delta^{n}\right)$ with $0 \leqslant \bar{z} \leqslant 1$. For all $z(x)$

$$
\begin{aligned}
\left\|\frac{\varphi^{n} \circ X^{n}-\varphi^{n}}{\triangle t}\left(\delta^{n}\right)^{1 / 2}\right\|^{2} & =\left(\Delta t^{-2}\right) \int_{\Omega}\left(\varphi^{n}(x)-\varphi^{n}\left(x-\frac{u_{h}^{n-1} \beta^{n-1}}{\phi(x)} \Delta t\right)\right)^{2} \delta^{n} d x \\
& =\left(\triangle t^{-2}\right) \int_{\Omega}\left(\int_{x-\frac{u_{h}^{n-1} \beta^{n-1}}{\phi(x)} \Delta t}^{x} \frac{\partial \varphi^{n}}{\partial z} d z\right)^{2} \delta^{n} d x
\end{aligned}
$$




$$
\begin{aligned}
& \leqslant\left(\triangle \mathrm{t}^{-2}\right) \int_{\Omega}\left(\int_{0}^{1} \frac{\partial \varphi^{\mathrm{n}}}{\partial z}\left(x-\frac{\mathrm{u}^{\mathrm{n}-1} \beta^{n-1}}{\phi} \Delta \mathrm{t} \bar{z}\right) \cdot \frac{\mathrm{u}^{\mathrm{n}-1} \beta^{n-1}}{\phi} \Delta \mathrm{td} \bar{z}\right)^{2} \delta^{\mathrm{n}} \mathrm{dx} \\
& \leqslant \mathrm{K} \int_{0}^{1} \int_{\Omega}\left|\nabla \varphi^{\mathrm{n}}\left(x-\frac{\mathrm{u}^{\mathrm{n}-1} \beta^{n-1}}{\phi} \Delta \mathrm{t} \bar{z}\right)\right|^{2} \cdot \delta^{\mathrm{n}} \mathrm{d} x \mathrm{~d} \bar{z} \\
& \leqslant \mathrm{~K} \int_{\Omega}\left|\nabla \varphi^{\mathrm{n}}(\mathrm{y})\right|^{2} \cdot \mathrm{d} \mathrm{y} \leqslant \mathrm{K}\left\|\nabla \varphi^{\mathrm{n}}(\mathrm{y})\right\|_{\mathrm{L}^{2},}^{2}
\end{aligned}
$$

then we get

$$
\mathrm{T}_{311} \leqslant \varepsilon \sum_{n=2}^{\mathrm{N}}\left\|\mathrm{d}_{\mathrm{t}} \xi_{\mathrm{c}}^{\mathrm{n}-1}\right\|_{\mathrm{L}^{2}}^{2} \Delta \mathrm{t}+\mathrm{K} \sum_{\mathrm{n}=2}^{\mathrm{N}}\left\|\nabla \xi_{\mathrm{c}}^{\mathrm{n}-1}\right\|_{\mathrm{L}^{2}}^{2} \Delta \mathrm{t}, \quad \mathrm{T}_{312} \leqslant \mathrm{~K} \sum_{\mathrm{n}=2}^{\mathrm{N}}\left(\left\|\nabla \xi_{\mathrm{c}}^{\mathrm{n}-1}\right\|_{\mathrm{L}^{2}}^{2}+\left\|\xi_{\mathrm{c}}^{\mathrm{n}-1}\right\|_{\mathrm{L}^{2}}^{2}\right) \triangle \mathrm{t} .
$$

For $T_{32}$, we have

$$
\begin{aligned}
\mathrm{T}_{32} & =\left(\frac{\left(\frac{\phi}{\beta_{h}^{N-1}} \xi_{c}^{\mathrm{N}-1}\right) \circ X^{N} \delta^{N}-\frac{\phi}{\beta_{h}^{N-1}} \xi_{c}^{N-1}}{\Delta t}, \xi_{c}^{N}\right) \\
& =\left(\frac{\left(\frac{\phi}{\beta_{h}^{N-1}} \xi_{c}^{N-1}\right) \circ X^{N} \delta^{N}}{\triangle t}, \xi_{c}^{N}\right)-\left(\frac{\left(\frac{\phi}{\beta_{h}^{N-1}} \xi_{c}^{N-1}\right) \circ X^{N} \delta^{N}}{\Delta t}, \xi_{c}^{N} \circ X^{N}\right) \\
& =\left(\left(\frac{\phi}{\beta_{h}^{N-1}} \xi_{c}^{N-1}\right) \circ X^{N} \delta^{N}, \frac{\xi_{c}^{N}-\xi_{c}^{N} \circ X^{N}}{\triangle t}\right) \\
& \leqslant K\left\|\left(\phi \xi_{c}^{N-1}\right) \circ X^{N}\left(\delta^{N}\right)^{1 / 2}\right\|_{L^{2}} \cdot\left\|\frac{\xi_{c}^{N}-\xi_{c}^{N-1} \circ X^{n}}{\triangle t}\left(\delta^{N}\right)^{1 / 2}\right\|_{L^{2}} \\
& \leqslant K\left\|\xi_{c}^{N-1}\right\|_{L^{2}}^{2}+\varepsilon\left\|\nabla \xi_{c}^{N}\right\|_{L^{2}}^{2}
\end{aligned}
$$

it implies that

$$
\mathrm{T}_{3} \leqslant \varepsilon \sum_{n=2}^{\mathrm{N}}\left\|\mathrm{d}_{\mathrm{t}} \xi_{\mathrm{c}}^{\mathrm{n}-1}\right\|_{\mathrm{L}^{2}}^{2} \Delta \mathrm{t}+\mathrm{K}\left\|\xi_{\mathrm{c}}^{\mathrm{N}-1}\right\|_{\mathrm{L}^{2}}^{2}+\varepsilon\left\|\nabla \xi_{\mathrm{c}}^{\mathrm{N}}\right\|_{\mathrm{L}^{2}}^{2}+\mathrm{K} \sum_{\mathrm{n}=2}^{\mathrm{N}}\left(\left\|\nabla \xi_{\mathrm{c}}^{\mathrm{n}-1}\right\|_{\mathrm{L}^{2}}^{2}+\left\|\xi_{\mathrm{c}}^{\mathrm{n}-1}\right\|_{\mathrm{L}^{2}}^{2}\right) \triangle \mathrm{t} .
$$

Now, we consider $T_{5}$. We have

$$
\begin{aligned}
T_{5} & =\sum_{n=1}^{N}\left(\frac{\left(\frac{\phi}{\beta_{h}^{n-1}} \zeta_{c}^{n-1}\right) \circ X^{n} \delta^{n}-\frac{\phi}{\beta_{h}^{n-1}} \zeta_{c}^{n-1}}{\Delta t}, d_{t} \xi_{c}^{n}\right) \Delta t \\
& =\sum_{n=1}^{N}\left(\frac{\left(\frac{\phi}{\beta_{h}^{n-1}} \zeta_{c}^{n-1}\right) \circ X^{n} \delta^{n}-\frac{\phi}{\beta_{h}^{n-1}} \zeta_{c}^{n-1}}{\Delta t}, \xi_{c}^{n}\right)-\left(\frac{\left(\frac{\phi}{\beta_{h}^{n-1}} \zeta_{c}^{n-1}\right) \circ X^{n} \delta^{n}-\frac{\phi}{\beta_{h}^{n-1}} \zeta_{c}^{n-1}}{\Delta t}, \xi_{c}^{n-1}\right) \\
& =\sum_{n=1}^{N}-\left(d_{t}\left(\left(\frac{\phi}{\beta_{h}^{n-1}} \zeta_{c}^{n-1}\right) \circ X^{n} \delta^{n}-\frac{\phi}{\beta_{h}^{n-1}} \zeta_{c}^{n-1}\right), \xi_{c}^{n-1}\right)+\left(\frac{\left(\frac{\phi}{\beta_{h}^{n-1}} \zeta_{c}^{N-1}\right) \circ X^{N} \delta^{N}-\frac{\phi}{\beta_{h}^{n-1}} \zeta_{c}^{N-1}}{\Delta t}, \xi_{c}^{N}\right) .
\end{aligned}
$$

With the same argument as $T_{3}$, we can get

$$
\begin{aligned}
\mathrm{T}_{5} & \leqslant \mathrm{~K} \sum_{n=1}^{\mathrm{N}}\left(\left\|\mathrm{d}_{\mathrm{t}} \zeta_{\mathrm{c}}^{\mathrm{n}-1}\right\|_{\mathrm{L}^{2}}^{2}+\left\|\nabla \zeta_{\mathrm{c}}^{\mathrm{n}-1}\right\|_{\mathrm{L}^{2}}^{2}+\left\|\zeta_{\mathrm{c}}^{\mathrm{n}}\right\|_{\mathrm{L}^{2}}^{2} \Delta \mathrm{t}+\mathrm{k}\left\|\zeta_{\mathrm{c}}^{\mathrm{N}-1}\right\|_{\mathrm{L}^{2}}^{2}+\varepsilon\left\|\nabla \xi_{\mathrm{c}}^{\mathrm{N}}\right\|_{\mathrm{L}^{2}}^{2}\right. \\
& \leqslant \mathrm{K}\left(\mathrm{h}_{\mathrm{c}}^{2 \mathrm{k}+2}+\sum_{\mathrm{n}=1}^{\mathrm{N}}\left\|\nabla \xi_{\mathrm{c}}^{\mathrm{n}-1}\right\|_{\mathrm{L}^{2}}^{2} \Delta \mathrm{t}\right)+\varepsilon\left\|\nabla \xi_{\mathrm{c}}^{\mathrm{N}}\right\|_{\mathrm{L}^{2}}^{2} .
\end{aligned}
$$

$\mathrm{T}_{4}$ can be easily bounded as follows

$$
\begin{aligned}
& \mathrm{T}_{4}=\sum_{n=1}^{\mathrm{N}}\left(\frac{\frac{\phi}{\beta_{h}^{n-1}} \zeta_{\mathrm{c}}^{\mathrm{n}}-\frac{\phi}{\beta_{\mathrm{h}}^{\mathrm{n}-1}} \zeta_{\mathrm{c}}^{\mathrm{n}-1}}{\triangle \mathrm{t}}, \mathrm{d}_{\mathrm{t}} \xi_{\mathrm{c}}^{\mathrm{n}}\right) \triangle \mathrm{t} \\
& \leqslant K \sum_{n=1}^{N}\left\|d_{t} \zeta_{c}^{n}\right\|_{L^{2}}^{2} \Delta t+\varepsilon \sum_{n=1}^{N}\left\|d_{t} \xi_{c}^{n}\right\|_{L^{2}}^{2} \triangle t \leqslant K\|c\|_{H^{1}\left(H^{2}\right)}^{2} h_{c}^{2 k+2}+\varepsilon \sum_{n=1}^{N}\left\|d_{t} \xi_{c}^{n}\right\|_{L^{2}}^{2} \triangle t .
\end{aligned}
$$


And it is easy to see

$$
\begin{aligned}
& \mathrm{T}_{6}=\sum_{n=1}^{N}\left(\nabla \cdot\left(\left(\mathbf{u}^{\mathrm{n}}-\mathbf{u}_{\mathrm{h}}^{\mathrm{n}-1}\right) \mathrm{c}^{\mathrm{n}}\right), \mathrm{d}_{\mathrm{t}} \xi_{\mathrm{c}}^{\mathrm{n}}\right) \triangle \mathrm{t} \\
& =\sum_{n=1}^{N}-\left(\mathbf{u}^{n}-\mathbf{u}_{h}^{n-1}, c^{n} \nabla d_{t} \xi_{c}^{n}\right) \triangle t \\
& \leqslant \sum_{n=1}^{N}\left\{\left|\left(\int_{t_{n-1}}^{t_{n}} \frac{\partial \mathbf{u}}{\partial t} d t \cdot c^{n}, \nabla d_{t} \xi_{c}^{n}\right)\right|+\left|\left(\left(a\left(c_{h}^{n}\right)-a\left(c_{h}^{n-1}\right)\right) \sigma^{n-1} \cdot c^{n-1}, \nabla d_{t} \xi_{c}^{n}\right)\right|\right. \\
& \left.+\left|\left(a\left(c_{h}^{n-1}\right) \xi_{\sigma}^{n-1} \cdot c^{n}, \nabla d_{t} \xi_{c}^{n}\right)\right|+\left|\left(a\left(c_{h}^{n-1}\right) \zeta_{\sigma}^{n-1} \cdot c^{n}, \nabla d_{t} \xi_{c}^{n}\right)\right|\right\} \triangle t \\
& \leqslant \mathrm{~K} \sum_{n=1}^{\mathrm{N}}\left\{\left\|\xi_{\mathrm{c}}^{\mathrm{n}-1}\right\|_{\mathrm{L}^{2}}^{2}+\left\|\zeta_{\mathrm{c}}^{\mathrm{n}-1}\right\|_{\mathrm{L}^{2}}^{2}+\left\|\xi_{\sigma}^{n-1}\right\|_{\mathrm{L}^{2}}^{2}+\left\|\zeta_{\sigma}^{n-1}\right\|_{\mathrm{L}^{2}}^{2}+\Delta \mathrm{t}\left\|\frac{\partial \mathbf{u}}{\partial \mathrm{t}}\right\|_{\mathrm{L}^{2}\left((0, \mathrm{~T}] ; \mathrm{L}^{2}\right)}^{2}\right\} \triangle \mathrm{t} \\
& +\varepsilon \sum_{n=1}^{\mathrm{N}}\left(\left\|\nabla \xi_{\mathrm{c}}^{n}\right\|_{\mathrm{L}^{2}}^{2}-\left\|\nabla \xi_{\mathrm{c}}^{\mathrm{n}-1}\right\|_{\mathrm{L}^{2}}^{2}\right) \\
& \leqslant K\left(h_{\sigma}^{2 r+2}+h_{c}^{2 k+2}\right)+K \sum_{n=1}^{N}\left(\left\|\xi_{c}^{n-1}\right\|^{2}+\left\|\xi_{\sigma}^{n-1}\right\|^{2}+\Delta t\left\|\frac{\partial \mathbf{u}}{\partial t}\right\|_{L^{2}\left((0, T] ; L^{2}\right)}^{2}\right) \Delta t \\
& +\varepsilon \sum_{n=1}^{\mathrm{N}}\left(\left\|\nabla \xi_{\mathrm{c}}^{\mathrm{n}}\right\|_{\mathrm{L}^{2}}^{2}-\left\|\nabla \xi_{\mathrm{c}}^{\mathrm{n}-1}\right\|_{\mathrm{L}^{2}}^{2}\right)
\end{aligned}
$$

and

$$
\mathrm{T}_{8} \leqslant \mathrm{~K} \sum_{n=1}^{\mathrm{N}}\left\|\tilde{\zeta}_{\mathrm{c}}^{n}\right\|_{\mathrm{L}^{2}}^{2} \Delta \mathrm{t}+\varepsilon \sum_{n=1}^{\mathrm{N}}\left\|\mathrm{d}_{\mathrm{t}} \xi_{\mathrm{c}}^{\mathrm{n}}\right\|_{\mathrm{L}^{2}}^{2} \Delta \mathrm{t} \leqslant \mathrm{K}\|\mathrm{c}\|_{\mathrm{H}^{1}\left(\mathrm{H}^{2}\right)}^{2} \mathrm{~h}_{\mathrm{c}}^{2 \mathrm{k}+2}+\varepsilon \sum_{\mathrm{n}=1}^{\mathrm{N}}\left\|\mathrm{d}_{\mathrm{t}} \xi_{\mathrm{c}}^{\mathrm{n}}\right\|_{\mathrm{L}^{2}}^{2} \Delta \mathrm{t} .
$$

Combining these above estimates, we can easily get the inequality (4.3).

\section{Error estimate for the flux}

Set $\xi_{\sigma}^{n}=\sigma_{h}^{n}-\Pi_{h} \sigma^{n}, \zeta_{\sigma}^{n}=\sigma^{n}-\Pi_{h} \sigma^{n}, \xi_{H}^{n}=H_{h}^{n}-Q_{h} H^{n}$, and $\zeta_{H}^{n}=H^{n}-Q_{h} H^{n}$. We have to estimate bounds of $\xi_{\sigma}$ and $\xi_{H}$, which satisfy the error residual equations:

$$
\begin{aligned}
\left(\frac{\alpha\left(c_{h}^{n}\right) \xi_{\sigma}^{n}-\alpha\left(c_{h}^{n-1}\right) \xi_{\sigma}^{n-1}}{\Delta t}, \mathbf{v}_{h}\right)+\left(B \nabla \cdot \xi_{\sigma}^{n}, \nabla \cdot \mathbf{v}_{h}\right) \\
=\left(\frac{\partial}{\partial t}(\alpha(c) \sigma)-\frac{\alpha\left(c^{n}\right) \sigma^{n}-\alpha\left(c^{n-1}\right) \sigma^{n-1}}{\triangle t}, \mathbf{v}_{h}\right)+\left(B \phi \eta\left(\frac{\partial c}{\partial t}-\frac{c^{n}-c^{n-1}}{\triangle t}\right), \nabla \cdot \mathbf{v}_{h}\right) \\
\quad+\left(\eta\left(\frac{\partial c}{\partial t}-\frac{c^{n}-c^{n-1}}{\triangle t}\right) \mathbf{e}_{3}, \mathbf{v}_{h}\right)+\left(\frac{\alpha\left(c_{h}^{n}\right) \zeta_{\sigma}^{n}-\alpha\left(c_{h}^{n-1}\right) \zeta_{\sigma}^{n-1}}{\triangle t}, \mathbf{v}_{h}\right) \\
\quad+\left(\frac{\left[\alpha\left(c^{n}\right)-\alpha\left(c_{h}^{n}\right)\right] \sigma^{n}-\left[\alpha\left(c^{n-1}\right)-\alpha\left(c_{h}^{n-1}\right)\right] \sigma^{n-1}}{\triangle t}, \mathbf{v}_{h}\right) \\
\quad+\left(B \nabla \cdot \zeta_{\sigma}^{n}, \nabla \cdot \mathbf{v}_{h}\right)+\left(B q^{n}\left(\beta_{h}^{n}-\beta^{n}\right), \nabla \cdot \mathbf{v}_{h}\right) \\
\quad+\left(B \phi \eta \frac{\zeta_{c}^{n}-\zeta_{c}^{n-1}}{\triangle t}, \nabla \cdot \mathbf{v}_{h}\right)-\left(B \phi \eta \frac{\xi_{c}^{n}-\xi_{c}^{n-1}}{\triangle t}, \nabla \cdot \mathbf{v}_{h}\right) \\
\quad+\left(\eta \frac{\zeta_{c}^{n}-\zeta_{c}^{n-1}}{\triangle t} \mathbf{e}_{3}, \mathbf{v}_{h}\right)-\left(\eta \frac{\xi_{c}^{n}-\xi_{c}^{n-1}}{\triangle t} \mathbf{e}_{3}, \mathbf{v}_{h}\right), \quad \forall \mathbf{v}_{h} \in V_{h}
\end{aligned}
$$


and

$$
\begin{aligned}
\left(\frac{\xi_{H}^{n}-\xi_{H}^{n-1}}{\triangle t}, w_{h}\right)= & \left(B q^{n}\left(\beta_{h}^{n}-\beta^{n}\right), w_{h}\right)-\left(B \nabla \cdot\left(\sigma_{h}^{n}-\sigma^{n}\right), w_{h}\right) \\
& +\left(B \phi \eta\left(\frac{\partial c}{\partial t}-\frac{c^{n}-c^{n-1}}{\triangle t}\right), w_{h}\right)+\left(B \phi \eta \frac{\zeta_{c}^{n}-\zeta_{c}^{n-1}}{\triangle t}, \nabla \cdot \mathbf{v}_{h}\right) \\
& -\left(B \phi \eta \frac{\xi_{c}^{n}-\xi_{c}^{n-1}}{\triangle t}, w_{h}\right), \quad \forall w_{h} \in \mathcal{W}_{h} .
\end{aligned}
$$

To obtain the error estimate for the flux, we also make other induction hypotheses as follows:

$$
\begin{gathered}
\max _{n}\left\|c_{h}^{n}\right\|_{\infty} \leqslant k h_{c}^{-\frac{1}{2}}\left[\frac{h_{c}^{\frac{3}{2}}}{\Delta t}\right]^{\frac{1}{2}}, \\
\max _{n}\left\|\xi_{\sigma}^{n}\right\|_{L^{2}}+\max _{n}\left\|\xi_{c}^{n}\right\|_{L^{2}}=o\left(\max \left(h_{\sigma}^{\frac{3}{2}}, h_{c}^{\frac{3}{2}}\right)\right) .
\end{gathered}
$$

From MCC-SMFE Algorithm, we can easily show that (5.3) and (5.4) hold when $n=0$. Now, we assume that (5.3) and (5.4) hold for $n=1,2, \ldots, N-1$, we have the following results.

Lemma 5.1. Assume that $\alpha, \alpha^{\prime}$, and $\alpha^{\prime \prime}$ are bounded, then the priori estimate

$$
\begin{aligned}
& \left(\frac{\left[\alpha\left(c^{n}\right)-\alpha\left(c_{h}^{n}\right)\right] \sigma^{n}-\left[\alpha\left(c^{n-1}\right)-\alpha\left(c_{h}^{n-1}\right)\right] \sigma^{n-1}}{\triangle t}, \mathbf{v}_{h}\right) \\
& \leqslant \\
& \quad K\left\{\left\|\xi_{c}^{n}\right\|_{L^{2}}^{2}+\left\|\xi_{c}^{n-1}\right\|_{L^{2}}^{2}+\left\|\xi_{c}^{n-2}\right\|_{L^{2}}^{2}+\left\|\xi_{\sigma}^{n-1}\right\|_{L^{2}}^{2}\right. \\
& \left.\quad+\left\|\xi_{\sigma}^{n-2}\right\|_{L^{2}}^{2}+\left\|\nabla \cdot \mathbf{v}_{h}\right\|_{L^{2}}^{2}+h_{c}^{2 k+2}+h_{\sigma}^{2 r+2}+(\triangle t)^{2}\right\}+\delta\left\|\mathbf{v}_{h}\right\|_{L^{2}}^{2}
\end{aligned}
$$

holds, for any $\mathbf{v}_{\mathrm{h}} \in \mathcal{V}_{\mathrm{h}}$.

Proof. Note that

$$
\begin{gathered}
\boldsymbol{\sigma}^{n-1}=\sigma^{n}-\int_{t_{n-1}}^{t_{n}} \frac{\partial \sigma}{\partial t} d t \\
\alpha\left(c^{n}\right)-\alpha\left(c^{n-1}\right)=\int_{0}^{1} \alpha^{\prime}\left(c^{n-1}+s\left(c^{n}-c^{n-1}\right)\right) d s\left(c^{n}-c^{n-1}\right), \\
\alpha\left(c_{h}^{n}\right)-\alpha\left(c_{h}^{n-1}\right)=\int_{0}^{1} \alpha^{\prime}\left(c_{h}^{n-1}+s\left(c_{h}^{n}-c_{h}^{n-1}\right)\right) d s\left(c_{h}^{n}-c_{h}^{n-1}\right) .
\end{gathered}
$$

So we have

$$
\begin{aligned}
& {\left[\alpha\left(c^{n}\right)-\alpha\left(c^{n-1}\right)\right]-\left[\alpha\left(c_{h}^{n}\right)-\alpha\left(c_{h}^{n-1}\right)\right]} \\
& \quad=\int_{0}^{1} \alpha^{\prime}\left(c_{h}^{n-1}+s\left(c_{h}^{n}-c_{h}^{n-1}\right)\right) d s\left(\eta_{c}^{n}-\eta_{c}^{n-1}\right)-\int_{0}^{1} \alpha^{\prime}\left(c_{h}^{n-1}+s\left(c_{h}^{n}-c_{h}^{n-1}\right)\right) d s\left(\xi_{c}^{n}-\xi_{c}^{n-1}\right) \\
& \quad+\int_{0}^{1}\left[\zeta_{c}^{n-1}+s\left(\zeta_{c}^{n}-\zeta_{c}^{n-1}\right)\right] \alpha^{\prime \prime} d s \int_{t_{n-1}}^{t_{n}} \frac{\partial c}{\partial t} d t-\int_{0}^{1}\left[\xi_{c}^{n-1}+s\left(\xi_{c}^{n}-\xi_{c}^{n-1}\right)\right] \alpha^{\prime \prime} d s \int_{t_{n-1}}^{t_{n}} \frac{\partial c}{\partial t} d t .
\end{aligned}
$$

Utilizing this equation, we can easily get

$$
\begin{aligned}
& \left(\frac{\left[\alpha\left(c^{\mathfrak{n}}\right)-\alpha\left(c_{h}^{n}\right)\right] \sigma^{n}-\left[\alpha\left(c^{n-1}\right)-\alpha\left(c_{h}^{n-1}\right)\right] \sigma^{n-1}}{\triangle t}, \mathbf{v}_{h}\right) \\
& \quad=\left(\frac{\left[\alpha\left(c^{n}\right)-\alpha\left(c_{h}^{n}\right)-\left(\alpha\left(c^{n-1}\right)-\alpha\left(c_{h}^{n-1}\right)\right)\right] \sigma^{n}}{\triangle t}, \mathbf{v}_{h}\right)+\left(\left[\alpha\left(c^{n-1}\right)-\alpha\left(c_{h}^{n-1}\right)\right] \frac{1}{\Delta t} \int_{t_{n-1}}^{t_{n}} \frac{\partial \sigma}{\partial t} d t, \mathbf{v}_{h}\right)
\end{aligned}
$$




$$
\begin{aligned}
= & \left(\int_{0}^{1} \alpha^{\prime}\left(c_{h}^{n-1}+s\left(c_{h}^{n}-c_{h}^{n-1}\right)\right) d s \sigma^{n} \frac{\zeta_{c}^{n}-\zeta_{c}^{n-1}}{\Delta t}, \mathbf{v}_{h}\right) \\
& -\left(\int_{0}^{1} \alpha^{\prime}\left(c_{h}^{n-1}+s\left(c_{h}^{n}-c_{h}^{n-1}\right)\right) d s \sigma^{n} \frac{\xi_{c}^{n}-\xi_{c}^{n-1}}{\Delta t}, \mathbf{v}_{h}\right) \\
& +\left(\int_{0}^{1}\left[\zeta_{c}^{n-1}+s\left(\zeta_{c}^{n}-\zeta_{c}^{n-1}\right)\right] \alpha^{\prime \prime} d s \frac{\sigma^{n}}{\Delta t} \int_{t_{n-1}}^{t_{n}} \frac{\partial c}{\partial t} d t, \mathbf{v}_{h}\right) \\
& -\left(\int_{0}^{1}\left[\xi_{c}^{n-1}+s\left(\xi_{c}^{n}-\xi_{c}^{n-1}\right)\right] \alpha^{\prime \prime} d s \frac{\sigma^{n}}{\triangle t} \int_{t_{n-1}}^{t_{n}} \frac{\partial c}{\partial t} d t, \mathbf{v}_{h}\right) \\
& +\left(\left[\alpha\left(c^{n-1}\right)-\alpha\left(c_{h}^{n-1}\right)\right] \frac{1}{\Delta t} \int_{t_{n-1}}^{t_{n}} \frac{\partial \sigma}{\partial t} d t, \mathbf{v}_{h}\right)=F_{1}+F_{2}+F_{3}+F_{4}+F_{5} .
\end{aligned}
$$

Using Lemma 4.1, we can derive

$$
\begin{aligned}
\mathrm{F}_{1}+\mathrm{F}_{3}+\mathrm{F}_{4}+\mathrm{F}_{5} \leqslant & K\left\{\left\|\xi_{\mathrm{c}}^{\mathrm{n}}\right\|_{\mathrm{L}^{2}}^{2}+\left\|\xi_{\mathrm{c}}^{\mathrm{n}-1}\right\|_{\mathrm{L}^{2}}^{2}+\left\|\xi_{\mathrm{c}}^{\mathrm{n}-2}\right\|_{\mathrm{L}^{2}}^{2}+\left\|\xi_{\sigma}^{\mathrm{n}-1}\right\|_{\mathrm{L}^{2}}^{2}\right. \\
& \left.+\left\|\xi_{\sigma}^{\mathrm{n}-2}\right\|_{\mathrm{L}^{2}}^{2}+\left\|\nabla \cdot \mathbf{v}_{\mathrm{h}}\right\|_{\mathrm{L}^{2}}^{2}+\mathrm{h}_{\mathrm{c}}^{2 \mathrm{k}+2}+\mathrm{h}_{\sigma}^{2 \mathrm{r}+2}+(\Delta \mathrm{t})^{2}\right\}+\delta\left\|\mathbf{v}_{\mathrm{h}}\right\|_{\mathrm{L}^{2}}^{2} .
\end{aligned}
$$

For $F_{2}$, we have

$$
\begin{aligned}
F_{2} & =\left(\frac{\phi}{\beta_{h}^{n-1}} \frac{\xi_{c}^{n}-\xi_{c}^{n-1}}{\Delta t}, \int_{0}^{1} \alpha^{\prime}\left(c_{h}^{n-1}+s\left(c_{h}^{n}-c_{h}^{n-1}\right)\right) d s \sigma^{n} \cdot v_{h} \beta_{h}^{n-1} / \phi\right) \\
& =\left(\frac{\phi}{\beta_{h}^{n-1}} \frac{\xi_{c}^{n}-\xi_{c}^{n-1}}{\Delta t}, R_{M}\left[\int_{0}^{1} \alpha^{\prime}\left(c_{h}^{n-1}+s\left(c_{h}^{n}-c_{h}^{n-1}\right)\right) d s \sigma^{n} \cdot \mathbf{v}_{h} \beta_{h}^{n-1} / \phi\right]\right) \\
& \leqslant K\left\{\left\|\xi_{c}^{n}\right\|_{L^{2}}^{2}+\left\|\xi_{c}^{n-1}\right\|_{L^{2}}^{2}+\left\|\xi_{\sigma}^{n-1}\right\|_{L^{2}}^{2}+\left\|\nabla \cdot \mathbf{v}_{h}\right\|_{L^{2}}^{2}+h_{c}^{2 k+2}+h_{\sigma}^{2 r+2}+(\Delta t)^{2}\right\}+\delta\left\|\mathbf{v}_{h}\right\|_{L^{2}}^{2},
\end{aligned}
$$

where $R_{M}$ is a weighted $L^{2}$-projection operator from $L^{2}(\Omega)$ onto $\mathcal{M}_{h}$ such that

$$
\left(\frac{\phi}{\beta_{h}^{n-1}}\left(z-\mathrm{R}_{M} z\right), z_{\mathrm{h}}\right)=0, \quad \forall z \in \mathrm{L}^{2}(\Omega), z_{\mathrm{h}} \in \mathcal{M}_{\mathrm{h}}
$$

Substituting (5.7) and (5.8) into (5.6), we get the estimate (5.5).

Lemma 5.2. Under the conditions of Lemmas 4.1 and 5.1, we have the following estimate

$$
\begin{aligned}
& \left(\frac{\alpha\left(c_{h}^{n}\right) \xi_{\sigma}^{n}-\alpha\left(c_{h}^{n-1}\right) \xi_{\sigma}^{n-1}}{\triangle t}, \mathbf{v}_{h}\right)+\left(B \nabla \cdot \xi_{\sigma}^{n}, \nabla \cdot \mathbf{v}_{h}\right) \\
& \leqslant K\left\{\left\|\xi_{c}^{n}\right\|_{L^{2}}^{2}+\left\|\xi_{c}^{n-1}\right\|_{L^{2}}^{2}+\left\|\xi_{c}^{n-2}\right\|_{L^{2}}^{2}+\left\|\xi_{\sigma}^{n-1}\right\|_{L^{2}}^{2}+\left\|\xi_{\sigma}^{n-2}\right\|_{L^{2}}^{2}+\left\|\nabla \cdot \mathbf{v}_{h}\right\|_{L^{2}}^{2}\right. \\
& \left.\quad+h_{\sigma}^{2 r+2}+h_{\sigma}^{2 r_{1}+2}+h_{c}^{2 k+2}+(\triangle t)^{2}\right\}+\delta\left\|\mathbf{v}_{h}\right\|_{L^{2}}^{2} .
\end{aligned}
$$

Proof. It is easily seen that

$$
\begin{aligned}
\left(\frac{\partial}{\partial t}\left(\alpha(c) \sigma^{n}\right)-\frac{\alpha\left(c^{n}\right) \sigma^{n}-\alpha\left(c^{n-1}\right) \sigma^{n-1}}{\triangle t}, \mathbf{v}_{h}\right) \\
+\left(B \phi \eta\left(\frac{\partial c}{\partial t}-\frac{c^{n}-c^{n-1}}{\triangle t}\right), \nabla \cdot \mathbf{v}_{h}\right)+\left(\eta\left(\frac{\partial c}{\partial t}-\frac{c^{n}-c^{n-1}}{\triangle t}\right) \mathbf{e}_{3}, \mathbf{v}_{h}\right) \\
\leqslant K\left\{\triangle t\left[\left\|\frac{\partial^{2} \alpha(c) \sigma}{\partial t^{2}}\right\|_{L^{2}\left(J^{n} ; L^{2}\right)}^{2}+\left\|\frac{\partial^{2} c}{\partial t^{2}}\right\|_{L^{2}\left(J^{n} ; L^{2}\right)}^{2}\right]+\left\|\nabla \cdot \mathbf{v}_{h}\right\|_{L^{2}}^{2}\right\}+\delta\left\|\mathbf{v}_{h}\right\|_{L^{2},}^{2}, \\
\left(B q^{n}\left(\beta_{h}^{n}-\beta^{n}\right), \nabla \cdot \mathbf{v}_{h}\right) \leqslant K\left\{\left\|\xi_{c}^{n}\right\|_{L^{2}}^{2}+\left\|\eta_{c}^{n}\right\|_{L^{2}}^{2}+\left\|\nabla \cdot \mathbf{v}_{h}\right\|_{L^{2}}^{2}\right\},
\end{aligned}
$$


and

$$
\left(\mathrm{B} \phi \eta \frac{\zeta_{\mathrm{c}}^{\mathrm{n}}-\zeta_{\mathrm{c}}^{\mathrm{n}-1}}{\triangle \mathrm{t}}, \nabla \cdot \mathbf{v}_{\mathrm{h}}\right)+\left(\eta \frac{\zeta_{\mathrm{c}}^{\mathrm{n}}-\zeta_{\mathrm{c}}^{\mathrm{n}-1}}{\triangle \mathrm{t}} \mathbf{e}_{3}, \mathbf{v}_{\mathrm{h}}\right) \leqslant \mathrm{K}\left\{\left\|\nabla \cdot \mathbf{v}_{\mathrm{h}}\right\|_{\mathrm{L}^{2}}^{2}+\mathrm{h}_{\mathrm{c}}^{2 \mathrm{k}+2}\right\}+\delta\left\|\mathbf{v}_{\mathrm{h}}\right\|_{\mathrm{L}^{2}}^{2} .
$$

As noted above, we know that

$$
\begin{aligned}
\alpha\left(c_{h}^{n-1}\right)= & \alpha\left(c_{h}^{n}\right)-\int_{0}^{1} \alpha^{\prime}\left(c_{h}^{n-1}+s\left(c_{h}^{n}-c_{h}^{n-1}\right)\right) d s\left(c_{h}^{n}-c_{h}^{n-1}\right) \\
= & \alpha\left(c_{h}^{n}\right)-\int_{0}^{1} \alpha^{\prime}\left(c_{h}^{n-1}+s\left(c_{h}^{n}-c_{h}^{n-1}\right)\right) d s\left(\xi_{c}^{n}-\xi_{c}^{n-1}\right) \\
& +\int_{0}^{1} \alpha^{\prime}\left(c_{h}^{n-1}+s\left(c_{h}^{n}-c_{h}^{n-1}\right)\right) d s\left(\zeta_{c}^{n}-\zeta_{c}^{n-1}\right)-\int_{0}^{1} \alpha^{\prime}\left(c_{h}^{n-1}+s\left(c_{h}^{n}-c_{h}^{n-1}\right)\right) d s \int_{t_{n-1}}^{t_{n}} \frac{\partial c}{\partial t} d t .
\end{aligned}
$$

So we have

$$
\begin{aligned}
&\left(\frac{\alpha\left(c_{h}^{n}\right) \zeta_{\sigma}^{n}-\alpha\left(c_{h}^{n-1}\right) \zeta_{\sigma}^{n-1}}{\triangle t}, \mathbf{v}_{h}\right) \\
&=\left(\alpha\left(c_{h}^{n}\right) \frac{\zeta_{\sigma}^{n}-\zeta_{\sigma}^{n-1}}{\Delta t}, \mathbf{v}_{h}\right)+\left(\int_{0}^{1} \alpha^{\prime}\left(c_{h}^{n-1}+s\left(c_{h}^{n}-c_{h}^{n-1}\right)\right) d s \zeta_{\sigma}^{n-1} \frac{\xi_{c}^{n}-\xi_{c}^{n-1}}{\triangle t}, \mathbf{v}_{h}\right) \\
& \quad-\left(\int_{0}^{1} \alpha^{\prime}\left(c_{h}^{n-1}+s\left(c_{h}^{n}-c_{h}^{n-1}\right)\right) d s \zeta_{\sigma}^{n-1} \frac{\zeta_{c}^{n}-\zeta_{c}^{n-1}}{\triangle t}, \mathbf{v}_{h}\right) \\
& \quad+\left(\int_{0}^{1} \alpha^{\prime}\left(c_{h}^{n-1}+s\left(c_{h}^{n}-c_{h}^{n-1}\right)\right) d s \zeta_{\sigma}^{n-1} \frac{1}{\triangle t_{n}} \int_{t_{n-1}}^{t_{n}} \frac{\partial c}{\partial t} d t, \mathbf{v}_{h}\right) \\
& \leqslant\left(\frac{\phi}{\beta_{h}^{n-1}} \frac{\xi_{c}^{n}-\xi_{c}^{n-1}}{\triangle t_{n}}, R_{M}\left[\int_{0}^{1} \alpha^{\prime}\left(c_{h}^{n-1}+s\left(c_{h}^{n}-c_{h}^{n-1}\right)\right) d s \zeta_{\sigma}^{n-1} \cdot \mathbf{v}_{h} \beta_{h}^{n-1} / \phi\right]\right) \\
&+K\left\{\frac{1}{\triangle t}\left\|\frac{\partial \zeta_{\sigma}}{\partial t}\right\|_{L^{2}\left(J^{n} ; L^{2}\right)}^{2}+\left\|\zeta_{\sigma}^{n-1}\right\|_{L^{2}}^{2}+\left\|\mathbf{v}_{h}\right\|_{L^{2}}^{2}\right\} \\
& \leqslant K\left\{\left\|\xi_{c}^{n-1}\right\|_{L^{2}}^{2}+\left\|\xi_{\sigma}^{n-1}\right\|_{L^{2}}^{2}+\left\|\nabla \cdot \mathbf{v}_{h}\right\|_{L^{2}}^{2}+h_{\sigma}^{2 r+2}+h_{c}^{2 k+2}+(\Delta t)^{2}\right\}+\delta\left\|\mathbf{v}_{h}\right\|_{L^{2}}^{2} .
\end{aligned}
$$

By Lemma 3.1 and the inverse property of the finite element space $\mathcal{V}_{h}$, we have the estimate

$$
\left(\mathrm{B} \nabla \cdot \zeta_{\sigma}^{\mathrm{n}}, \nabla \cdot \mathbf{v}_{\mathrm{h}}\right) \leqslant \mathrm{Kh}_{\sigma}\left\|\nabla \cdot \zeta_{\sigma}^{\mathrm{n}}\right\|_{\mathrm{L}^{2}}\left\|\nabla \cdot \mathbf{v}_{\mathrm{h}}\right\|_{\mathrm{L}^{2}} \leqslant \mathrm{Kh}_{\sigma}^{2 \mathrm{r}_{1}+2}+\delta\left\|\nabla \cdot \mathbf{v}_{\mathrm{h}}\right\|_{\mathrm{L}^{2}}^{2} .
$$

Utilizing the similar technique as in (5.8), we can get the following inequality

$$
\begin{aligned}
& \left|\left(B \phi \eta \frac{\xi_{c}^{n}-\xi_{c}^{n-1}}{\triangle t}, \nabla \cdot \mathbf{v}_{h}\right)\right|+\left|\left(\eta \frac{\xi_{c}^{n}-\xi_{c}^{n-1}}{\Delta t} \mathbf{e}_{3}, \mathbf{v}_{h}\right)\right| \\
& \quad \leqslant K\left\{\left\|\xi_{c}^{n-1}\right\|_{L^{2}}^{2}+\left\|\xi_{\sigma}^{n-1}\right\|_{L^{2}}^{2}+\left\|\nabla \cdot \mathbf{v}_{h}\right\|_{L^{2}}^{2}+h_{\sigma}^{2 r+2}+h_{c}^{2 k+2}+(\triangle t)^{2}\right\}+\delta\left\|\mathbf{v}_{h}\right\|_{L^{2}}^{2} .
\end{aligned}
$$

Substituting these estimates into (5.1), we complete the proof of Lemma 5.2.

Lemma 5.3. Under the conditions of Lemma 5.2, we have the estimate

$$
\begin{aligned}
& \sum_{n=1}^{N}\left\|d_{t} \xi_{\sigma}^{n}\right\|_{L^{2}}^{2} \Delta t+\left\|\nabla \cdot \xi_{\sigma}^{N}\right\|_{L^{2}}^{2} \\
& \quad \leqslant K\left\{\sum_{n=1}^{N-1}\left[\left\|\xi_{\sigma}^{n}\right\|_{L^{2}}^{2}+\left\|\xi_{c}^{n}\right\|_{L^{2}}^{2}+\left\|\nabla \cdot \xi_{\sigma}^{n}\right\|_{L^{2}}^{2}\right] \Delta t+h_{\sigma}^{2 r+2}+h_{\sigma}^{2 r_{1}+2}+h_{c}^{2 k+2}+(\Delta t)^{2}\right\} .
\end{aligned}
$$


Proof. Taking $\mathbf{v}_{\mathrm{h}}=\mathrm{d}_{\mathrm{t}} \xi_{\sigma}^{n}$ in (5.9), and noting that

$$
\left(\frac{\alpha\left(c_{h}^{n}\right) \xi_{\sigma}^{n}-\alpha\left(c_{h}^{n-1}\right) \xi_{\sigma}^{n-1}}{\Delta t}, \xi_{\sigma}^{n}\right)=\left(\alpha\left(c_{h}^{n}\right) d_{t} \xi_{\sigma}^{n}, d_{t} \xi_{\sigma}^{n}\right)+\left(d_{t}\left(\alpha\left(c_{h}^{n}\right)\right) \xi_{\sigma}^{n-1}, d_{t} \xi_{\sigma}^{n}\right),
$$

and

$$
\begin{aligned}
\left(B \nabla \cdot \xi_{\sigma}^{n}, \nabla \cdot d_{t} \xi_{\sigma}^{n}\right) & \geqslant \frac{1}{2} d_{t}\left(B \nabla \cdot \xi_{\sigma}^{n}, \nabla \cdot \xi_{\sigma}^{n}\right)-\frac{1}{2}\left(d_{t} B \nabla \cdot \xi_{\sigma}^{n-1}, \nabla \cdot \xi_{\sigma}^{n-1}\right) \\
& \geqslant \frac{1}{2} d_{t}\left(B \nabla \cdot \xi_{\sigma}^{n}, \nabla \cdot \xi_{\sigma}^{n}\right)-K\left\|\nabla \cdot \xi_{\sigma}^{n-1}\right\|^{2} .
\end{aligned}
$$

Under the inductive induction hypothesis (5.4), using the similar technique as in (5.8) and Lemma 4.1, we get

$$
\begin{aligned}
& {\left[\left(\alpha\left(c_{h}^{n}\right) d_{t} \xi_{\sigma}^{n}, d_{t} \xi_{\sigma}^{n}\right)-\left(d_{t}\left(\alpha\left(c_{h}^{n}\right)\right) \xi_{\sigma}^{n-1}, d_{t} \xi_{\sigma}^{n}\right)\right]+\frac{1}{\Delta t}\left(\left\|\nabla \cdot \xi_{\sigma}^{n}\right\|^{2}-\left\|\nabla \cdot \xi_{\sigma}^{n-1}\right\|^{2}\right)-K\left\|\nabla \cdot \xi_{\sigma}^{n-1}\right\|} \\
& \leqslant \\
& \quad K\left\{\left\|\xi_{c}^{n}\right\|_{L^{2}}^{2}+\left\|\xi_{\sigma}^{n}\right\|_{L^{2}}^{2}+\left\|\xi_{c}^{n-1}\right\|_{L^{2}}^{2}+\left\|\xi_{c}^{n-2}\right\|_{L^{2}}^{2}+\left\|\xi_{\sigma}^{n-1}\right\|_{L^{2}}^{2}+\left\|\xi_{\sigma}^{n-2}\right\|_{L^{2}}^{2}\right. \\
& \left.\quad+\left\|\nabla \cdot \mathbf{v}_{h}\right\|_{L^{2}}^{2}+h_{\sigma}^{2 r+2}+h_{\sigma}^{2 r_{1}+2}+h_{c}^{2 k+2}+(\triangle t)^{2}\right\}+\delta\left\|\mathbf{v}_{h}\right\|_{L^{2}}^{2} .
\end{aligned}
$$

Multiplying (5.11) by $2 \triangle t$ and summing it over $n$, for sufficiently small $\delta$, we get the estimate (5.10).

\section{The proof of Theorem 2.1}

Now, we can complete the proof of Theorem 2.1.

Proof. Note the fact that

$$
\left\|\varphi^{\mathrm{N}}\right\|_{\mathrm{L}^{2}}^{2}-\left\|\varphi^{0}\right\|_{\mathrm{L}^{2}}^{2} \leqslant \mathrm{~K} \sum_{n=1}^{\mathrm{N}}\left\|\varphi^{\mathrm{n}}\right\|_{\mathrm{L}^{2}}^{2} \Delta \mathrm{t}+\varepsilon \sum_{n=1}^{\mathrm{N}}\left\|\mathrm{d}_{\mathrm{t}} \varphi^{\mathrm{n}}\right\|_{\mathrm{L}^{2}}^{2} \triangle \mathrm{t} .
$$

Using Lemmas 4.1 and 5.3, we can get

$$
\begin{aligned}
& \left\|\xi_{\sigma}^{\mathrm{N}}\right\|_{\mathrm{L}^{2}}^{2}+\left\|\xi_{\mathrm{c}}^{\mathrm{N}}\right\|_{\mathrm{L}^{2}}^{2}+\left\|\nabla \cdot \xi_{\sigma}^{\mathrm{N}}\right\|_{\mathrm{L}^{2}}^{2}+\left\|\nabla \xi_{\mathrm{c}}^{\mathrm{N}}\right\|_{\mathrm{L}^{2}}^{2}+\sum_{n=1}^{\mathrm{N}}\left\|\mathrm{d}_{\mathrm{t}} \xi_{\mathrm{c}}^{\mathrm{n}}\right\|_{\mathrm{L}^{2}}^{2} \Delta \mathrm{t}+\sum_{n=1}^{\mathrm{N}}\left\|\mathrm{d}_{\mathrm{t}} \xi_{\sigma}^{\mathrm{n}}\right\|_{\mathrm{L}^{2}}^{2} \Delta \mathrm{t} \\
& \quad \leqslant \mathrm{K}\left\{\Delta \mathrm{t} \sum_{\mathrm{n}=1}^{\mathrm{N}-1}\left[\left\|\xi_{\sigma}^{\mathrm{n}}\right\|_{\mathrm{L}^{2}}^{2}+\left\|\xi_{\mathrm{c}}^{\mathrm{n}}\right\|_{\mathrm{L}^{2}}^{2}+\left\|\nabla \cdot \xi_{\sigma}^{\mathrm{n}}\right\|_{\mathrm{L}^{2}}^{2}+\left\|\nabla \xi_{\mathrm{c}}^{\mathrm{n}}\right\|_{\mathrm{L}^{2}}^{2}\right]+\mathrm{h}_{\sigma}^{2 \mathrm{r}+2}+\mathrm{h}_{\sigma}^{2 r_{1}+2}+\mathrm{h}_{\mathrm{c}}^{2 \mathrm{k}+2}+(\Delta \mathrm{t})^{2}\right\} .
\end{aligned}
$$

Applying the discrete Gronwall's inequality, we have

$$
\begin{aligned}
& \left\|\xi_{\sigma}^{\mathrm{N}}\right\|_{\mathrm{L}^{2}}^{2}+\left\|\xi_{\mathrm{c}}^{\mathrm{N}}\right\|_{\mathrm{L}^{2}}^{2}+\left\|\nabla \cdot \xi_{\sigma}^{\mathrm{N}}\right\|_{\mathrm{L}^{2}}^{2}+\left\|\nabla \xi_{\mathrm{c}}^{\mathrm{N}}\right\|_{\mathrm{L}^{2}}^{2}+\Delta \mathrm{t} \sum_{i=1}^{\mathrm{N}}\left[\left\|\mathrm{d}_{\mathrm{t}} \xi_{\sigma}^{\mathrm{n}}\right\|_{\mathrm{L}^{2}}^{2}+\left\|\mathrm{d}_{\mathrm{t}} \xi_{\mathrm{c}}^{\mathrm{n}}\right\|_{\mathrm{L}^{2}}^{2}\right] \\
& \leqslant \mathrm{K}\left\{\mathrm{h}_{\sigma}^{2 r+2}+\mathrm{h}_{\sigma}^{2 r_{1}+2}+\mathrm{h}_{\mathrm{c}}^{2 \mathrm{k}+2}+(\Delta \mathrm{t})^{2}\right\} .
\end{aligned}
$$

It is clear that the optimal error estimate (6.2) is derived under the inductive hypotheses (4.1), (5.3), and (5.4). Now we have to check it when $n=N$. Under the condition (2.8), for the integers $r, k, r_{1}>0$, using (6.2) we know that

$$
\begin{aligned}
\left\|\mathbf{u}_{h}^{N}\right\|_{\infty}=\left\|a\left(c_{h}^{N}\right) \boldsymbol{\sigma}_{h}^{N}\right\|_{\infty} & \leqslant K\left\{\left\|\Pi_{h} \sigma^{N}\right\|_{\infty}+\left\|\xi_{\sigma}^{N}\right\|_{\infty}\right\} \\
& \leqslant K\left\{\left\|\Pi_{h} \sigma^{N}\right\|_{\infty}+h_{\sigma}^{-\frac{3}{2}}\left\|\xi_{\sigma}^{N}\right\|_{L^{2}}\right\}
\end{aligned}
$$




$$
\begin{aligned}
& \leqslant K\left\{\left\|\Pi_{h} \sigma^{n}\right\|_{\infty}+h_{\sigma}^{-\frac{3}{2}}\left(h_{\sigma}^{r+1}+h_{\sigma}^{r_{1}+1}+h_{c}^{k+1}+\Delta t\right)\right\} \leqslant K h_{\sigma}^{-\frac{1}{2}}\left[\frac{h_{\sigma}^{\frac{3}{2}}}{\Delta t}\right]^{\frac{1}{2}}, \\
\left\|c_{h}^{N}\right\|_{\infty} & \leqslant K\left\{\left\|P_{h} c^{N}\right\|_{\infty}+\left\|\xi_{c}^{N}\right\|_{\infty}\right\} \\
& \leqslant K\left\{\left\|P_{h} c^{N}\right\|_{\infty}+h_{c}^{-\frac{3}{2}}\left\|\xi_{c}^{N}\right\|_{L^{2}}\right\} \\
& \leqslant K\left\{\left\|P_{h} c^{n}\right\|_{\infty}+h_{c}^{-\frac{3}{2}}\left(h_{\sigma}^{r+1}+h_{\sigma}^{r_{1}+1}+h_{c}^{k+1}+\triangle t\right)\right\} \leqslant K h_{c}^{-\frac{1}{2}}\left[\frac{h_{c}^{\frac{3}{2}}}{\Delta t}\right]^{\frac{1}{2}}
\end{aligned}
$$

and

$$
\left\|\xi_{\mathrm{c}}^{\mathrm{N}}\right\|_{\mathrm{L}^{2}}+\left\|\xi_{\sigma}^{\mathrm{N}}\right\|_{\mathrm{L}^{2}} \leqslant \mathrm{~K}\left\{\mathrm{~h}_{\sigma}^{\mathrm{r}+1}+\mathrm{h}_{\sigma}^{\mathrm{r}_{1}+1}+\mathrm{h}_{\mathrm{c}}^{\mathrm{k}+1}+\Delta \mathrm{t}\right\} .
$$

So, we know that the inductive hypotheses (4.1), (5.3), and (5.4) are true for $n=N$.

Finally, we consider the bound of $\xi_{H}$. Taking $w_{h}=\xi_{H}^{n}$ in (5.2) and using the estimate (6.2), we can easily get

$$
\max _{n}\left\|\xi_{H}^{n}\right\|_{L^{2}}^{2} \leqslant K\left\{h_{c}^{2 k+2}+h_{\sigma}^{2 r+2}+h_{\sigma}^{2 r_{1}}+h_{H}^{2 l+2}+(\triangle t)^{2}\right\} \text {. }
$$

This ends the proof of Theorem 2.1.

\section{Acknowledgment}

The authors would like to express their sincere thanks to the editor and the reviewer for their very helpful comments and suggestions, which greatly improved the quality of this paper.

This work was supported partially by the National Natural Science Foundation of China (11401588), the Natural Science Foundation of Shandong Province (ZR2014AQ005) and the Fundamental Research Funds for the Central Universities (17CX02043).

\section{References}

[1] R. A. Adams, Sobolev spaces, Pure and Applied Mathematics, Academic Press [A subsidiary of Harcourt Brace Jovanovich, Publishers], New York-London, (1975). 2

[2] P. F. Anderson Jr., H. O. White, J. W. Mercer, P. S. Huyakorn, Numerical modeling of groundwater flow and saltwater transport in northern pinellas country, Southwest Florida Water Management Distric, Florida, (1985). 1, 1

[3] J. B. Bell, C. N. Dawson, G. R. Shubin, An unsplit, higher order Godunov method for scalar conservation laws in multiple dimensions, J. Comput. Phys., 74 (1988), 1-24. 1

[4] M. A. Cella, T. F. Russell, I. Herrera, R. E. Ewing, An Eulerian-Lagrangian localized adjoint method for the advectiondiffusion equation, Adv. Water Resour., 13 (1990), 187-206. 1

[5] P. G. Ciarlet, The finite element method for elliptic problems, Studies in Mathematics and its Applications, NorthHolland Publishing Co., Amsterdam-New York-Oxford, (1978). 3, 3, 3.1

[6] H. J. Diersch, Finite element modelling of recirculating density-driven saltwater intrusion processes in groundwater, Adv. Water Resour., 11 (1988), 25-43. 1

[7] J. Douglas Jr., T. F. Russell, Numerical methods for convection-dominated diffusion problems based on combining the method of characteristics with finite element or finite difference procedures, SIAM J. Numer. Anal., 19 (1982), 871-885. 1

[8] A. D. Gupta, P. N. Yapa, Saltwater encroachment in an aquifer: A case study, Water Resour. Res., 18 (1982), 546-556. 1

[9] P. S. Huyakorn, P. F. Anderson, J. W. Mercer, H. O. White Jr., Saltwater intrusion in aquifers: Development and testing of a three dimensional finite element model, Water Resour. Res., 23 (1987), 293-312. 1

[10] C. Johnson, Streamline diffusion methods for problems in fluid mechanics, Finite Elem. Fluids, 6 (1986), 251-261. 1

[11] X.-M. Lian, H.-X. Rui, A discontinuous Galerkin method combined with mixed finite element for seawater intrusion problem, J. Syst. Sci. Complex., 23 (2010), 830-845. 1, 2.3

[12] H.-X. Rui, M. Tabata, A mass-conservative characteristic finite element scheme for convection-diffusion problems, J. Sci. Comput., 43 (2010), 416-432. 1, 2.1

[13] G. Segol, G. F. Pinder, W. G. Gray, A Galerkin finite element technique for calculating the transient position of the saltwater front, Water Resour. Res., 11 (1975), 343-347. 1 
[14] H. Wang, An optimal-order error estimate for a family of ELLAM-MFEM approximations to porous medium flow, SIAM J. Numer. Anal., 46 (2008), 2133-2152. 1

[15] Y.-Q. Xue, C.-H. Xie, J.-C. Wang, Study on joint-surface of saltwater and freshwater in seawater intrusion problem, Nanjing University Press, Nanjing, (1991), 43-94. 1

[16] D.-P. Yang, Analysis of least-squares mixed finite element methods for nonlinear nonstationary convection-diffusion problems, Math. Comp., 69 (2000), 929-963. 1

[17] D.-P. Yang, A splitting positive definite mixed element method for miscible displacement of compressible flow in porous media, Numer. Methods Partial Differential Equations, 17 (2001), 229-249. 1, 3.1

[18] Y.-R. Yuan, N. Du, Y.-J. Han, Careful numerical simulation and analysis of migration-accumulation of Tanhai Region, Appl. Math. Mech., 26 (2005), 741-752. 1

[19] Y.-R. Yuan, D. Liang, H.-X. Rui, Characteristics-finite element methods for seawater intrusion numerical simulation and theoretical analysis, Acta Math. Appl. Sinica (English Ser.), 14 (1998), 11-23. 1, 1, 2.3

[20] Y.-R. Yuan, D. Liang, H.-X. Rui, Numerical method and simulation of seawater intrusion and protection projects, Chinese J. Comput. Phys., 18 (2001), 562-567. 1

[21] Y.-R. Yuan, D. Liang, H.-X. Rui, Predicting the consequences of seawater intrusion and protection projects, Appl. Math. Mech., 22 (2001), 1291-1300. 1

[22] Y.-R. Yuan, D. Liang, H.-X. Rui, G.-H. Wang, Characteristic-difference methods for the numerical simulation of sea water intrusion and optimal order $l^{2}$ error estimates, (Chinese) Acta Math. Appl. Sinica, 19 (1996), 395-404. 1

[23] Y.-R. Yuan, H.-X. Rui, D. Liang, C.-F. Li, The theory and application of upwind finite difference fractional steps procedure for seawater intrusion, Int. J. Geosci., 3 (2012), 972-991. 1

[24] Z.-Y. Zhang, The alternating-direction schemes and numerical analysis for the three-dimensional seawater intrusion simulation, Acta Math. Appl. Sin. Engl. Ser., 18 (2002), 389-404. 1

[25] J.-S. Zhang, H. Guo, A split least-squares characteristic mixed element method for nonlinear nonstationary convectiondiffusion problem, Int. J. Comput. Math., 89 (2012), 932-943. 1

[26] J.-S. Zhang, D.-P. Yang, A fully-discrete splitting positive definite mixed finite element scheme for compressible miscible displacement in porous media, J. Shandong Univ. Nat. Sci., 41 (2006), 1-10.

[27] J.-S. Zhang, D.-P. Yang, A splitting positive definite mixed element method for second-order hyperbolic equations, Numer. Methods Partial Differential Equations, 25 (2009), 622-636.

[28] J.-S. Zhang, D.-P. Yang, S.-Q. Shen, J. Zhu, A new MMOCAA-MFE method for compressible miscible displacement in porous media, Appl. Numer. Math., 80 (2014), 65-80.

[29] Y.-Z. Zhang, J.-S. Zhang, The splitting mixed element method for parabolic equation and its application in chemotaxis model, Appl. Math. Comput., 313 (2017), 287-300.

[30] J.-S. Zhang, J. Zhu, R.-P. Zhang, Characteristic splitting mixed finite element analysis of Keller-Segel chemotaxis models, Appl. Math. Comput., 278 (2016), 33-44. 1 